\title{
Social Energy as the Driving Force behind Crowdfunding-Analysis and Classification of Selected Attributes
}

\author{
Agnieszka Szewczyk *(D) and Zbigniew Stempnakowski *(D)
}

check for

updates

Citation: Szewczyk, A.;

Stempnakowski, Z. Social Energy as

the Driving Force behind

Crowdfunding-Analysis and

Classification of Selected Attributes

Energies 2021, 14, 6062. https://

doi.org/10.3390/en14196062

Academic Editors:

Wadim Strielkowski and

Wen-Hsien Tsai

Received: 11 August 2021

Accepted: 18 September 2021

Published: 23 September 2021

Publisher's Note: MDPI stays neutral with regard to jurisdictional claims in published maps and institutional affiliations.

Copyright: (C) 2021 by the authors. Licensee MDPI, Basel, Switzerland. This article is an open access article distributed under the terms and conditions of the Creative Commons Attribution (CC BY) license (https:/ / creativecommons.org/licenses/by/ $4.0 /)$.
Institute of Economics and Finance, Department of Information Society and Applied Informatics, University of Szczecin, 71-101 Szczecin, Poland

* Correspondence: agnieszka.szewczyk@usz.edu.pl (A.S.); zbigniew.stempnakowski@usz.edu.pl (Z.S.)

\begin{abstract}
Crowdfunding has been part of sharing economy for a few decades. Research into crowdfunding usually concerns its relation to sustainable development and corporate social responsibility, influencing investment decisions, psychological, organizational and financial aspects or the success evaluation of individual campaigns or platforms. Recent research includes empirical case studies, e.g., evaluation of the crowdfunding for minor coarse cereal products through evolutionary game analysis, or the impact of crowdfunding on the willingness to visit local festivals. There is, however, no comprehensive cross-sectional approach to crowdfunding attributes that considers data from various categories. The authors aimed to analyze selected attributes of crowdfunding, namely project categories, the number of backers, campaign duration and profitability, and to classify these attributes. A novelty is the use of the term "social energy" in a different sense than previously found in the scientific literature. An original algorithm for analyzing and classifying selected crowdfunding attributes and measuring the relationship between them was also used. The value of the article is also the practical application of its results. The findings have a practical outcome: they can be used by project creators, potential backers, investors and owners of crowdfunding platforms.
\end{abstract}

Keywords: crowdfunding; classification analysis of attributes; social energy; sharing (collaborative) economy; classification methods and criteria; research areas of crowdfunding; Kickstarter platform

\section{Introduction}

The term "energy" is usually identified with the field of physics, and in this field, it is used in the sense of electricity. Similar terms are also chemical, thermal, nuclear and solar energy, depending on its source.

Other approaches to the term "energy", used in the colloquial sense, are vital forces and readiness for work of a given organism. It is said, "he is bursting with energy", or "he was gone by energy, because he does not continue this action". This interpretation comes from Chinese literature. Here, the source of energy is food, and the unit of measurement is the calorie, as well as proper motivation, praise and the like.

Energy is also one of the basic terms of classical metaphysics, closely related to movement and to the act whose source is Aristotle's philosophy [1].

Different from the above is the term "social energy". This is clearly a practical approach, because there are no studies or research results related to this term in the literature, and which would be a form of references to various types of applications of social renewable energy choices. In this aspect, the following terms are used: associations or foundations supporting people in need, or a network of enterprises implementing new models of services in the field of public utility, especially for socially excluded people.

In this article, the authors use the term "social energy" in a completely different, innovative sense, as a driving force, i.e., mobilization to financially support various projects. On the surface, the term "crowd energy" may seem unambiguous, but it has only a psychological aspect (for example, duplication of behavior patterns) that is also important in crowdfunding, but not as the only one. It is also about the motives for joining the 
financing, from charity, devoid of expecting anything in return, to the desire to achieve certain profits or, at least, the so-called rewards. As a new, original solution, in addition to the novel use of the term "social energy", the authors bring analysis and classification of selected crowdfunding attributes and measurement of the relationships between them.

Launching such a "giant" as crowdfunding becomes possible with the participation of hundreds or even millions of people; that is, it requires great social energy. Hence, the presented research certainly fits into the area of sustainable energy and society.

The relationship between sustainable energy, economy and society was interpreted and studied in many different aspects. Some of the papers viewed the above issues from a global, international perspective; for example, there are the papers entitled "Externalities of power generation in Visegrad countries and their integration through support of renewables" [2], "Energy Efficiency in the Long-Run in the Selected European Countries" [3] and "Ranking of Baltic States on progress towards the main energy security goals of European energy union strategy". [4]

Many authors have tackled the highly important problems of energy security, which is of fundamental importance in the sustainable development of all areas of life, for example, social, economic and cultural. As a result of this research, a number of cross-sectional papers were created; for example, there are the papers entitled "Estimating the interrelation between energy security and macroeconomic factors in European countries" [5] and "Social and Economic Properties of the Energy Markets". [6]

There are also interesting publications analyzing the threats to society resulting from the energy production processes; for example, there are the papers entitled "Decomposition analysis of the impact of economic growth on ammonia and nitrogen oxides emissions in the European Union" [7] and "Sustainable agriculture and energy in the U.S.: A link between ethanol production and the acreage for corn" [8], as well as "Sustainable supply chain of the biomass cluster as a factor for preservation and enhancement of forests" [9]. The latter paper is extremely valuable, as it raises the issues of the need to care for the national welfare of forests.

However, this article highlights the role of social energy (crowd energy) that "drives" crowdfunding, the essence of which is discussed below.

Crowdfunding has become one of the most popular ways to raise money for certain goals, projects or events by natural persons. It is based on soliciting for many small amounts of money from many individuals, who are called the community or-by other authors-the crowd [10-13].

The participation of hundreds of thousands or even millions of people in crowdfunding campaigns after mere twenty-something years since the first campaign was launched shows the scale of this phenomenon. It has already gained its place in economic theory, where it is presented as a form of the so-called sharing (collaborative) economy [14-17]. The financial perspective on crowdfunding confirms its global and financially powerful nature. This is evidenced by the following sample data from 2020 (fundly.com):

- Global amount collected by crowdfunding-USD 34 billion,

- Crowdfunding's contribution to the global economy-USD 65 billion,

- Global average success rate of crowdfunding campaigns-22.4\%,

- Percentage of US consumers aware of crowdfunding-64\%,

- Value of average donation to a crowdfunding campaign-\$99,

- $78 \%$ of successful campaigns exceed their fundraising target.

What is more, it has been predicted that, by 2025, the global crowdfunding market will almost triple. 
It has already been observed that, since the outbreak of the COVID-19 pandemic, the crowdfunding market has been growing significantly, especially crowdfunding with donations that are aimed at supporting and aiding communities, people and organizations in their battle with the pandemic. For instance, in April 2020, Facebook launched "Facebook Fundraiser", a platform where people can raise funds for charity to help others during the pandemic.

Moreover, in April 2020, various crowdfunding platforms in India raised over INR 100 crores to provide financial aid to people in need. Those Internet crowdfunding campaigns help immobile migrants, everyday workers, transgender communities, circus artists, Uber drivers, Swiggy delivery workers, rural craftsmen, dancers and freelancers during the pandemic.

It is also expected that reward-based crowdfunding will experience significant growth $[18,19]$. It frequently involves a business (usually a start-up) raising capital before starting its activity through an online platform and offering investors gifts or "extras" for their financial contribution. This is considered a pre-sale where individual persons (projects) or companies obtain funding from capital donors who then receive a non-monetary reward. This allows companies to get involved in building networks and strive to get something which can be used later. This type of crowdfunding is usually appropriate for early-stage companies or for a specific project. For example, the Australian start-up Okra Solar offers its "smart plugand-play controller", which makes it easier for neighbors to share energy. The company launched a reward-based crowdfunding campaign to raise funds to build the prototype and raised over $\$ 45,000$. The prototype worked, and the company subsequently obtained high government subsidies for serial production.

Other similarly successful projects were "The Sun Juicer" Ultralight Parabolic Solar Cooker, where 240 backers pledged \$19,152, and Nomad Energy—Radically Sustainable Energy Drink, with 257 backers pledging $\$ 16,678$.

Unfortunately, due to the lack of reliable, complete and up-to-date information, some projects do not reach their financial goals, even though they concerned the production, distribution and other aspects of broadly understood issues of energy or sustainable development. These include projects such as "Long Range Electric Motorcycle (Development/Prototype)", "La ciudad consciente: A tiny house on wheels community" and "RNWBL: Goods That Fund Clean Energy Projects".

The above, albeit very general, look at the current and future state of crowdfunding gives the basis for the conclusion that the world is dealing with an extensive and developing phenomenon. Hence, the study and analysis of its attributes in various respects becomes the duty of many scientists [20,21]. It is difficult to grasp and examine or analyze the entirety of crowdfunding range, taking into account its specificity and various attributes. However, one can try to pick out its selected features and group them in order to easier analyze macrodata.

The aim of this paper is to analyze selected attributes of crowdfunding: project categories, the number of people backing the projects, campaign duration, project profitability and rewards offered, and providing their mutual classification, i.e., creating classes of attributes that are similar to each other. The starting point of the algorithm is the so-called royalty, i.e., a measure of the closeness centrality (similarity) of elements. It was assumed that, due to the large variety and frequency of features, the method that takes into account two aspects is appropriate, which allows for the inclusion of two basic classification criteria, i.e., closeness and importance (weights). The created attribute classes allow for further cross-classification.

It should be noted that applying the classification method is justified by the specificity of the problem studied and the large amount of source data within the area of each attribute. The following research hypotheses were adopted:

- Project categories in crowdfunding contain similar elements, and it is possible to group them according to the adopted attributes and determine their weights; 
- $\quad$ Created classes are subject to another classification in terms of pairs (so-called tandems) and the strength of their correlation, i.e., the degree of interactions or lack thereof, can be defined.

The results of the research can be translated into practice and used by project creators, people considering financing their projects, including investors and owners of crowdfunding platforms.

It is worth emphasising that this practical aspect of the research is crucial. The need to conduct the study has been confirmed by reviewing academic literature and conducting a diagnostic survey among a randomly selected group of respondents. Both project creators and their potential backers expressed a lot of doubts, and there was a lack of access to full information necessary to make the right decision, e.g., which category the project should be put, how long the campaign should last, how many backers can be counted on or how to calculate the amount of the planned and necessary amount of money. Therefore, they can learn how to operate more effectively in the crowdfunding process and lead the project to success (also financial).

The main idea and importance of the presented research boils down to the right choice of crowdfunding attributes, capturing their interdependence, similarity and impact force, which enables a new, comprehensive, multifaceted movement in the maze of information about projects submitted for funding.

The proposed approach which utilizes the method of classifying categories and attributes, as well as the research results contribute to the current state of knowledge in the area of crowdfunding.

The article is organized as follows:

It begins with an introduction which emphasizes the importance of the topic, specifies the purpose of the article and its significance, and presents the hypotheses to be verified. The structure of the article is also presented. Then, a literature review of the subject in several specified areas is provided in a separate section. In the light of this analysis, the need for research provided for the purpose of the study is emphasized. The next section of the article contains the research methodology and information on the data used, included in the form of tables. The attribute classification method and statistical methods were used to determine the strength of attribute interactions. The developed algorithm takes as its starting point the so-called royalty, i.e., a measure of closeness (similarity) of components. It was assumed that, due to the large variety and frequency of features, the method that takes into account two aspects is appropriate, as it allows us to include two basic classification criteria: closeness and importance (weights). The created attribute classes were the basis for further cross-classification.

Then the results of the research were placed in a series of matrices and chains of the obtained classes along with their interpretation. The next part of the article is a discussion on the results and their interpretation in the perspective of the discussed research and the working hypotheses formulated in the introduction.

In reference to the aim of the article, the main parts of the research are cited, and the practical significance of the results, significantly broadening the horizon of information on crowdfunding mechanisms, is emphasized. Conclusions are extracted from the discussion and form the last part of the article. They present the degree of verification of the research hypotheses and the achievement of the aim of the article, as well as the main conclusions resulting from the conducted research. Research limitations are highlighted, potential weaknesses are identified and their importance is commented upon in relation to the overall interpretation of the results. The directions of future crowdfunding research are also suggested.

\section{Literature Review}

The analysis of the literature published especially in recent years has allowed to identify several areas of crowdfunding research. 
The first area includes a series of publications discussing the role of crowdfunding in achieving sustainable development and, particularly, a sustainable society. Some of the papers are reviews that show the relationship and effects of these elements. Examples of such a synthetic approach include Heidingsfelder and Beckmann [22], Testa et al. [23], Motylska-Kuzma [24] and Martínez-Climent et al. [25]. On the other hand, there is also research on selected aspects of crowdfunding affecting sustainable development, for example, the paper by Wehnert et al. [26] that analyzed the success of crowdfunding as a signal to increase trust in sustainable product goals, and the article by Liang et al. [27] that presented research on the impact of information description on the success of crowdfunding in a sustainable economy. The perspective of information communication that is outlined by these authors is also interesting. The presentation of the place of crowdfunding in sustainable retail and the formulation of the theoretical framework of success criteria by Konhäusner [28] is also noteworthy, as well as the paper by Jelinčić and Šveb [29] that shows the relationship between financial sustainability of cultural heritage based on an overview of crowdfunding in Europe.

The second research area of crowdfunding covers issues related to its impact on investments and the condition of companies' finances. Several significant publications should be mentioned here: Rossi et al. [30] discussed investment-based crowdfunding in the aspect of cross-platform analysis, Dilger et al. [31] assessed the crowdfunding potential for business model innovation of energy co-operatives, Wallmeroth et al. [32] discussed venture capital and crowdfunding of entrepreneurial ventures, Ter Wal et al. [33] presented issues of equity retention and social network theory in equity crowdfunding, Chu et al. [34] discussed open innovation in crowdfunding and Miglo [35] discussed some of the crowdfunding patterns of entrepreneurial firms in Canada.

Relatively few publications compare and analyze crowdfunding platforms. The paper by Rossi and Vismara [36] deserves attention for showing what crowdfunding platforms do and comparing investment platforms in Europe. Yu et al. [37] presented a specialized paper which used an interesting tool to assess the crowdfunding platform of minor coarse cereal products through evolutionary game analysis. Kang and Kim [38] answered the question of who can survive on an ICT-based crowdfunding platform, and Flórez-Parra et al. [39] discussed the role of a collective platform in strengthening economic and sustainable projects.

The most interesting papers are the ones in which crowdfunding is analyzed as part of behavioral economics, psychology, and sociology. The authors rightly see the need for behavioral research, especially the motivation of both investors and people co-financing various projects. The crowd syndrome has already been emphasized in this study, because it is the size of the reaction, attitude, reasons for joining the crowdfunding "machine" that determines the final success of the campaigns of given projects. Many works in this area deserve special attention.

Bretschneider and Leimeister [40] wrote interestingly about creating incentives for funding in reward-based crowdfunding systems; Pérez et al. [41] conducted an exploratory study on donation intentions to rural development; Zhang et al. [42] concentrated on the consumer's intent to purchase crowdfunding products or services in terms of costs and benefits; Kim and Hall [43] wondered if analyzing types of co-creation and crowdfunding can predict donor behavior and build an extended model of goal-directed behavior; Miglo [44] studied crowdfunding in a competitive environment; Seo and Park [45] analyzed the impact of other people's participation in investments on the psychological value of crowdfunding projects. On the other hand, Song and Tian [46] quoted the statements of managers and assessed customer involvement in crowdfunding; and Rodriguez-Ricardo et al. [47] emphasized that trust, altruism and a sense of internal control affect the motivation to participate in crowdfunding. The role of trust and investor intentions is also the focal point of the paper by Alharbey and Van Hemmen [48]. Mutual altruism and legitimacy in donation-based crowdfunding are also emphasized by Khurana [49], who cited evidence from India. A fairly original approach to crowdfunding was presented by Mohammadi 
and Shafi [50], whose paper analyzed the donation patterns of equity investors by gender. The results of a survey on motivations for equity-based crowdfunding in an emerging market were presented by Wasiuzzaman et al. [51] and answered the question of what institutional factors drive crowdfunding volumes can be found in the work of Kukk and Laidroo [52]. Kim et al. [53] presented an interesting study on behavioral influences on crowdfunding initiatives, emphasizing the importance of personality and subjective wellbeing. Yang et al. [54], based on the crowdfunding investment intentions model, showed the prospects of social exchange and the prospects of customer value.

Publications on broadly understood rewards for backers can also be placed in the field of behavioral economics. Virtually every project, goal or event for which funds are raised offers rewards and their aim is to encourage donations and promote a given initiative. The subject of incentives has been raised by relatively few authors and is most often associated with research on the success of a given campaign; for example Dikaputra et al. [55] analyzed the success factors of reward-based campaigns, using the so-called multi-theory approach. Comeig et al. [56] emphasized the importance of rational "herding" in reward-based crowdfunding. On the other hand, a comparative study of China and the United Kingdom conducted by Usman et al. [57] was designed with regard to the influence of signals on investment decisions.

Scientific research on crowdfunding in terms of description and correlation of financial success was conducted by Sauermann et al. [58] and Hörisch [59], who analyzed crowdfunding of environmental projects. Similar research on the success of distribution network campaigns can be found in the collective paper by Ter Wal et al. [60]. Usman et al. [61] tried to answer the question whether the role of the media and previous successes of the campaign's founder mitigate the problem of information asymmetry. The issue of what determines success on Kickstarter, with the emphasis on the role of anonymity and team, has been discussed by Ullah and Zhou [62], and empirical research determining the key success factors of a crowdfunding campaign of a given project was published by Fernandez-Blanco et al. [63].

A clearly behavioral approach was adopted in the article by Schraven et al. [64], called "Predictions of Crowdfunding Campaign Success: The Influence of First Impressions on Accuracy and Positivity". Venturelli et al. [65] also took on a behavioral perspective when investigating the relationship between gender and ethnic origin of the person submitting the project and its backers.

The research studies of Chen [66] (on the impact of competition and incentive design on the performance of crowdfunding projects on the example of independent films) and von Selasinsky and Lutz [67] (on the influence of pro-social and pro-environmental orientation) are more specialized studies on the impact of crowdfunding.

The above literature review allows for a conclusion that the interest of academics in the subject of crowdfunding seen from various perspectives is still significant, which can certainly be justified by the fact that this type of financing is developing dynamically. It takes different forms, which is reflected in research targeting large areas and models for donation, rewards, loans and equity. Therefore, the issue of continuing research remains relevant and worthy of attention.

\section{Materials and Methods}

The study was based on data downloaded from webrobots.io, which provides indexing and scraping services for websites. The robot crawls on websites and collects data from selected crowdfunding platforms in the format of ready-made CSV files. This indexing is carried out once a month, and this study was based on data collected for the Kickstarter platform on 17 June 2021.

The choice of Kickstarter as a data source can be explained by its leading position and wide popularity, as it appears in the five hundred most searched sites in the world, and it has brought success to over 205,000 projects since its inception. 
The data provided by webrobots.io are part of a public "raw" dataset that has also been used by other authors, for example, Reference [63]. There are data on over 31,000 campaigns in the analyzed set, of which over 20,000 were successful (Table 1).

Table 1. Breakdown of projects according to their status.

\begin{tabular}{cc}
\hline Project Status & Number of Campaigns \\
\hline Canceled & 1609 \\
\hline Failed & 7799 \\
\hline Live & 1209 \\
\hline Successful & 20,604 \\
\hline
\end{tabular}

Source: webrobots.io/kickstarter-datasets, data from 17 June 2021.

In the obtained project dataset, the following project attributes were selected for detailed research, taking into account data availability:

- $\quad$ Category - a group of subjects which the projects belong to;

- Number of people supporting the project;

- Campaign duration-calculated in days as the difference between the end date and the start date of the project;

- Project profitability, as the sum of collected donations;

- $\quad$ Rewards for supporters, offered by project creators.

When analyzing the first four attributes, no problems were encountered in identifying or systematizing them according to the adopted measures, which were quite obvious: subject, number of people, time and financial value. However, the attribute of "rewards" turned out to be impossible to assess unambiguously due to the great variety of the rewards. Other problems encountered when trying to analyze rewards were varied time periods in which they were offered or the fact that the number of rewards sometimes was limited and sometimes was not. All in all, rewards showed practically no repeatability in projects.

An additional problem was the fact that one of the most popular rewards-a discount on the purchase of the project result-was offered at almost every possible percentage and it most often depended on the amount of the donation. Moreover, the information about rewards appears and disappears from the offer according to unknown rules or rewards are turned into other rewards, such as a discount voucher to purchase two or more products, a digital product, a personalized thank-you for the supporter posted in the media, a design creator's handshake, a T-shirt, a mug or another gadget. The variety of currencies in the projects is also a big obstacle. The problem of rewards, in a very fragmentary scope, is illustrated in Table 2, which presents three randomly selected projects from different categories.

Taking the above factors into account, it was decided to exclude the "reward" attribute from further analyses and research, because any simplifications such as merely dividing rewards into discounts, gadgets, honorable mentions, etc., would make the credibility of such research-and thus its results in this respect-doubtful.

Data on the four attributes to be processed in the subsequent stages of the analysis and classification were specially prepared for the purposes of the methodology presented below:

- The names of the main and detailed categories were established by extracting these names from the general fields of the source data (Table 3);

- The ranges of the values of the attributes were specified-the number of backers, campaign duration and profitability-by analyzing various distributions of their values many times (the list of categories is presented in Table 4). 
Table 2. Sample rewards for 3 selected projects in selected categories.

\begin{tabular}{|c|c|c|c|}
\hline Project Category & Rewards Project 1 & Rewards Project 2 & Rewards Project 3 \\
\hline music & $\begin{array}{l}\$ 239 \text { for } 20 \% \text { off; } \$ 269 \text { for a } \\
10 \% \text { discount }\end{array}$ & $\begin{array}{l}\$ 69+\text { product; } \$ 79+\text { product; } \$ 99+ \\
\text { accessories; } \$ 345+\text { a few products }\end{array}$ & $\begin{array}{l}\$ 104 \text { for } 30 \% \text { discount; } \$ 179 \text { for } 40 \% \\
\text { discount; } \$ 246 \text { for } 45 \% \text { discount on } \\
3 \text { products }\end{array}$ \\
\hline wearables & $\begin{array}{l}\$ 899 \text { for } 27 \% \text { discount; } \$ 1048 \\
\text { for } 24 \% \text { discount }\end{array}$ & $\begin{array}{c}\$ 69 \text { for } 50 \% \text { discount; } \$ 79 \text { for } 39 \% \\
\text { discount; } \$ 148 \text { for } 42 \% \text { discount (on } \\
2 \text { products); } \$ 209 \text { for } 45 \% \text { discount } \\
\text { (on } 3 \text { products) }\end{array}$ & $\begin{array}{l}\$ 129 \text { for } 48 \% \text { discount; } \$ 256 \text { for } 48 \% \\
\text { discount (on } 2 \text { products); } \$ 384 \text { for } \\
48 \% \text { discount (on } 3 \text { products) }\end{array}$ \\
\hline art & $\begin{array}{l}\$ 6 \text { for a } 25 \% \text { discount; } \$ 21 \text { for } \\
30 \% \text { discount (set); } \$ 70 \text { for } \\
22 \% \text { discount (another set) }\end{array}$ & $\begin{array}{c}\text { EUR5 (digital product); EUR10 } \\
\text { (5 digital products); EUR30 (physical } \\
\text { product); EUR60 (full edition) }\end{array}$ & $\begin{array}{l}£ 100 \text { for } 33 \% \text { discount (on a } \\
\text { product); } £ 100 \text { for } 33 \% \text { discount (on } \\
\text { another product); }\end{array}$ \\
\hline graphic novels & $\begin{array}{l}\$ 25 \text { for } 16 \% \text { discount; } \$ 40 \text { for } \\
20 \% \text { discount (on another } \\
\text { product); } \$ 40 \text { for } 20 \% \text { discount } \\
\text { (product and merchandise); }\end{array}$ & $\begin{array}{l}\$ 75 \text { for a set; } \$ 20 \text { for a gadget; } \$ 25 \\
\text { for another gadget }\end{array}$ & $\begin{array}{l}\quad \$ 10 \text { for } 33 \% \text { discount (digital } \\
\text { product); } \$ 25 \text { for a product; } \$ 30 \text { for } \\
\text { a set of digital products; } \$ 150 \text { for a } \\
\text { collector's set }\end{array}$ \\
\hline photography & $\begin{array}{l}\$ 10 \text { support; } \$ 75 \text { first release; } \\
\$ 175 \text { box; } \$ 250 \text { set + extras; } \\
\$ 325-5 \text { copies of the product }\end{array}$ & $\begin{array}{c}\text { CAD68 product; CAD124-2 copies } \\
\text { of the product; CAD282-5 copies of } \\
\text { the product }\end{array}$ & $\begin{array}{l}\text { CHF44 product; CHF88-2 products; } \\
\text { CHF319-set; 365CHF-set + extras }\end{array}$ \\
\hline
\end{tabular}

Source: kickstarter.com, data from 17 June 2021.

Table 3. Summary of general source data.

\begin{tabular}{|c|c|c|c|c|c|}
\hline $\begin{array}{c}\text { Main } \\
\text { Category }\end{array}$ & $\begin{array}{l}\text { Detailed } \\
\text { Category }\end{array}$ & $\begin{array}{l}\text { Number of } \\
\text { Campaigns }\end{array}$ & $\begin{array}{c}\text { Number of } \\
\text { Backers }\end{array}$ & $\begin{array}{c}\text { Average } \\
\text { Campaign } \\
\text { Duration in Days }\end{array}$ & $\begin{array}{c}\text { Campaign } \\
\text { Profitability in } \\
\text { USD }\end{array}$ \\
\hline \multirow[t]{14}{*}{ art } & art & 938 & 95,553 & 24 & $4,911,459.55$ \\
\hline & ceramics & 256 & 29,058 & 29 & $2,653,205.10$ \\
\hline & conceptual art & 494 & 35,415 & 29 & $2,198,793.39$ \\
\hline & digital art & 152 & 11,527 & 26 & $562,192.97$ \\
\hline & illustration & 658 & 125,899 & 26 & $7,617,559.68$ \\
\hline & installations & 7 & 1110 & 29 & $188,940.77$ \\
\hline & mixed media & 92 & 6717 & 26 & $410,450.14$ \\
\hline & painting & 54 & 7761 & 30 & $891,525.83$ \\
\hline & performance art & 14 & 2540 & 01 & $531,851.32$ \\
\hline & public art & 25 & 2286 & 30 & $297,294.11$ \\
\hline & Sculpture & 33 & 3271 & 27 & $351,546.65$ \\
\hline & social practice & 20 & 1378 & 30 & $158,321.30$ \\
\hline & Textiles & 23 & 4047 & 29 & $251,218.17$ \\
\hline & video art & 8 & 637 & 05 & $66,423.69$ \\
\hline comics & graphic novels & 2338 & 669,144 & 29 & $34,782,426.91$ \\
\hline \multirow[t]{5}{*}{ dance } & dance & 1367 & 90,446 & 23 & $7,302,683.56$ \\
\hline & performances & 1626 & 100,678 & 23 & $8,606,656.83$ \\
\hline & residencies & 127 & 6419 & 20 & $554,838.90$ \\
\hline & spaces & 93 & 10,074 & 22 & $1,043,785.50$ \\
\hline & workshops & 59 & 3169 & 25 & $282,334.26$ \\
\hline
\end{tabular}


Table 3. Cont.

\begin{tabular}{|c|c|c|c|c|c|}
\hline $\begin{array}{c}\text { Main } \\
\text { Category }\end{array}$ & $\begin{array}{l}\text { Detailed } \\
\text { Category }\end{array}$ & $\begin{array}{l}\text { Number of } \\
\text { Campaigns }\end{array}$ & $\begin{array}{c}\text { Number of } \\
\text { Backers }\end{array}$ & $\begin{array}{c}\text { Average } \\
\text { Campaign } \\
\text { Duration in Days }\end{array}$ & $\begin{array}{c}\text { Campaign } \\
\text { Profitability in } \\
\text { USD }\end{array}$ \\
\hline fashion & jewelry & 616 & 50,181 & 21 & $4,568,444.84$ \\
\hline \multirow[t]{3}{*}{ film and video } & narrative film & 2257 & 487,842 & 26 & $47,507,147.04$ \\
\hline & romance & 82 & 6077 & 22 & $566,799.42$ \\
\hline & science fiction & 412 & 85,221 & 23 & $7,506,190.07$ \\
\hline \multirow[t]{8}{*}{ games } & games & 51 & 35,348 & 19 & $2,462,546.05$ \\
\hline & gaming hardware & 262 & 257,645 & 24 & $30,413,630.32$ \\
\hline & live games & 10 & 1229 & 31 & $109,664.97$ \\
\hline & mobile games & 5 & 2113 & 12 & $92,548.07$ \\
\hline & playing cards & 168 & 64,514 & 21 & $4,897,754.09$ \\
\hline & puzzles & 24 & 7798 & 21 & $517,103.64$ \\
\hline & tabletop games & 1352 & $1,393,194$ & 16 & $113,999,051.57$ \\
\hline & video games & 122 & 206,962 & 23 & $9,827,824.51$ \\
\hline music & classical music & 1937 & 158,512 & 29 & $13,928,401.89$ \\
\hline \multirow[t]{7}{*}{ photography } & animals & 149 & 30,943 & 24 & $3,424,682.11$ \\
\hline & fine art & 708 & 49,860 & 23 & $4,723,761.69$ \\
\hline & nature & 242 & 23,957 & 25 & $2,435,551.70$ \\
\hline & people & 250 & 21,417 & 26 & $1,694,268.07$ \\
\hline & photobooks & 1058 & 168,445 & 26 & $16,364,353.13$ \\
\hline & photography & 434 & 58,047 & 27 & $9,147,290.89$ \\
\hline & places & 127 & 11,804 & 25 & $791,674.10$ \\
\hline \multirow[t]{3}{*}{ publishing } & poetry & 709 & 46,635 & 26 & $2,394,154.92$ \\
\hline & radio and podcasts & 570 & 152,192 & 27 & $7,335,097.90$ \\
\hline & translations & 49 & 7639 & 30 & $504,963.28$ \\
\hline technology & wearables & 626 & 563,938 & 30 & $83,124,663.17$ \\
\hline
\end{tabular}

Source: webrobots.io/kickstarter-datasets, data from 17 June 2021.

Table 4. List of categories and their weights.

\begin{tabular}{cccccc}
\hline Category & Name & Weight & Category & Name & Weight \\
\hline graphic novels & K1 & 2338 & playing cards & K22 & 168 \\
\hline narrative film & K2 & 2257 & digital art & K23 & 152 \\
\hline classical music & K3 & 1937 & animals & K24 & 149 \\
\hline Performances & K4 & 1626 & places & K25 & 127 \\
\hline Dance & K5 & 1367 & video games & K26 & 122 \\
\hline tabletop games & K6 & 1352 & spaces & K27 & 93 \\
\hline photobooks & K7 & 1058 & mixed media & K28 & 92 \\
\hline art & K8 & 938 & romance & K29 & 82 \\
\hline poetry & K9 & 709 & workshops & K30 & 59 \\
\hline fine art & K10 & 708 & painting & K31 & 54 \\
\hline
\end{tabular}


Table 4. Cont.

\begin{tabular}{cccccc}
\hline Category & Name & Weight & Category & Name & Weight \\
\hline illustration & K11 & 658 & games & K32 & 51 \\
\hline wearables & K12 & 626 & translations & K33 & 49 \\
\hline jewelry & K13 & 616 & sculpture & K34 & 33 \\
\hline radio and podcasts & K14 & 570 & puzzles & K35 & 24 \\
\hline conceptual art & K15 & 494 & textiles & K36 & 23 \\
\hline photography & K16 & 434 & social practice & K37 & 20 \\
\hline science fiction & K17 & 412 & performance art & K38 & 14 \\
\hline gaming hardware & K18 & 262 & live games & K39 & 10 \\
\hline ceramics & K19 & 256 & video art & K40 & 8 \\
\hline people & K20 & 250 & installations & K41 & 7 \\
\hline nature & K21 & 242 & mobile games & K42 & 5 \\
\hline
\end{tabular}

Source: authors' own compilation based on webrobots.io/kickstarter-datasets, data from 17 June 2021.

It must be emphasized that when choosing only one platform to analyze, there is no need to systematize terminology, which would have to take place when examining multiple platforms, since practically each of them uses different (although often similar) nomenclature of categories or their internal structure. The study focuses only on those projects which were successful, thereby eliminating those campaigns which were canceled by the platform or by the creator, ongoing projects or failed ones. As a result, a dataset with 20,604 highly representative records was obtained.

The following tables contain the input data used in the study (Tables 5-7).

Ranges P1 to P41 represent the tiers in the attribute of the number of backers. The tier changes every 50 supporters and the last one represents the number of above 2000 people.

Ranges P1 to P31 represent the tiers in the attribute of the campaign duration. The tier changes every 2 days and the last one represents the duration of over 60 days.

Ranges P1 to P41 represent the tiers in the attribute of project's profitability. The tier changes every $\$ 1000$, and the last one represents the amount of over $\$ 40,000$. The amounts in US dollars were taken directly from the source databases.

The numerical values presented above were obtained by processing the source data in accordance with the established value ranges of the attributes. The "side" of the tables includes detailed project categories $(\mathrm{K} 1, \ldots, \mathrm{K} 42)$, sorted by decreasing weights, i.e., the number of projects in a given category. The "head" of the table shows ranges of the attributes: $(\mathrm{P} 1, \ldots, \mathrm{Pn})$. If in a given category there are projects within specified ranges, the number of these projects is placed at the intersection of the row and the column.

After the introductory stage, i.e., analyzing the crowdfunding attributes and preparing research data, the actual classification procedure can begin. First, it must be determined which classification method is adequate, that is, which best distinguishes various limitations or specific conditioning of the datasets.

In the general theoretical papers dedicated to the issues of classification, two basic concepts are present. The first is mainly represented by set theorists and mathematical logicians. According to the theory of Batley [68], classification understood as a whole is a "creation of the mind", in accordance with a certain accepted agreement. The existence of this whole raises many doubts about the structure of dependence on the mind that shapes it. The practical application of this concept causes many difficulties. 
Table 5. Numerical input data in terms of the number of backers (categories/ranges).

\begin{tabular}{|c|c|c|c|c|c|c|c|c|c|c|c|c|c|c|c|c|c|c|c|c|c|c|c|c|c|c|c|c|c|c|c|c|c|c|c|c|c|c|c|c|c|}
\hline Ranges & P1 & P2 & P3 & P4 & P5 & P6 & P7 & P8 & P9 & P10 & P11 & P12 & P13 & P14 & P15 & P16 & P17 & P18 & P19 & P20 & P21 & P22 & P23 & P24 & P25 & P26 & P27 & P28 & P29 & P30 & P31 & P32 & P33 & P34 & P35 & P36 & P37 & P38 & P39 & $\mathrm{P} 40$ & $\mathbf{P 4 1}$ \\
\hline \multicolumn{42}{|l|}{ Category } \\
\hline K1 & 440 & 207 & 115 & 55 & 37 & 28 & 14 & 10 & 6 & 5 & 3 & 4 & 2 & 0 & 2 & 3 & 2 & 0 & 3 & 0 & 0 & 0 & 0 & 0 & 1 & 0 & 0 & 0 & 0 & 0 & 0 & 0 & 0 & 0 & 0 & 1 & 0 & 0 & 0 & 0 & 0 \\
\hline K2 & 108 & 64 & 42 & 18 & 10 & 4 & 1 & 2 & 0 & 0 & 2 & 1 & 0 & 0 & 0 & 0 & 0 & 0 & 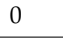 & 0 & 0 & 1 & 0 & 0 & 0 & 0 & 0 & 0 & 0 & 0 & 0 & 1 & 0 & 0 & 0 & 0 & 0 & 0 & 0 & 0 & 2 \\
\hline K4 & 82 & 34 & 17 & 8 & 5 & 2 & 0 & 1 & 0 & 1 & 1 & 0 & 0 & 0 & 1 & 0 & 0 & 0 & 0 & 0 & 0 & 0 & 0 & 0 & 0 & 0 & 0 & 0 & 0 & 0 & 0 & 0 & 0 & 0 & 0 & 0 & 0 & 0 & 0 & 0 & 0 \\
\hline K5 & 233 & 143 & 93 & 45 & 26 & 30 & 16 & 12 & 5 & 9 & 4 & 1 & 7 & 4 & 6 & 0 & 3 & 1 & 1 & 2 & 1 & 1 & 0 & 0 & 0 & 1 & 1 & 0 & 0 & 1 & 0 & 0 & 2 & 1 & 0 & 1 & 0 & 0 & 1 & 1 & 6 \\
\hline K6 & 2 & 2 & 0 & 1 & 0 & 1 & 0 & 0 & 0 & 0 & 1 & 0 & 0 & 0 & 0 & 0 & 0 & 0 & 0 & 0 & 0 & 0 & 0 & 0 & 0 & 0 & 0 & 0 & 0 & 0 & 0 & 0 & 0 & 0 & 0 & 0 & 0 & 0 & 0 & 0 & 0 \\
\hline K7 & 50 & 22 & 10 & 5 & 0 & 3 & 0 & 1 & 0 & 0 & 0 & 0 & 0 & 0 & 1 & 0 & 0 & 0 & 0 & 0 & 0 & 0 & 0 & 0 & 0 & 0 & 0 & 0 & 0 & 0 & 0 & 0 & 0 & 0 & 0 & 0 & 0 & 0 & 0 & 0 & 0 \\
\hline K9 & 4 & 4 & 1 & 0 & 1 & 1 & 1 & 0 & 1 & 0 & 0 & 0 & 0 & 0 & 1 & 0 & 0 & 0 & 0 & 0 & 0 & 0 & 0 & 0 & 0 & 0 & 0 & 0 & 0 & 0 & 0 & 0 & 0 & 0 & 0 & 0 & 0 & 0 & 0 & 0 & 0 \\
\hline K10 & 12 & 12 & 2 & 4 & 0 & 0 & 1 & 0 & 2 & 0 & 0 & 0 & 0 & 0 & 0 & 0 & 0 & 0 & 0 & 0 & 0 & 0 & 0 & 0 & 0 & 0 & 0 & 0 & 0 & 0 & 0 & 0 & 0 & 0 & 0 & 0 & 0 & 0 & 0 & 0 & 0 \\
\hline K11 & 14 & 2 & 1 & 1 & 0 & 1 & 1 & 0 & 0 & 0 & 0 & 0 & 0 & 0 & 0 & 0 & 0 & 0 & 0 & 0 & 0 & 0 & 0 & 0 & 0 & 0 & 0 & 0 & 0 & 0 & 0 & 0 & 0 & 0 & 0 & 0 & 0 & 0 & 0 & 0 & 0 \\
\hline K12 & 8 & 5 & 2 & 2 & 1 & 2 & 0 & 1 & 1 & 0 & 0 & 0 & 0 & 0 & 0 & 0 & 0 & 0 & 0 & 0 & 0 & 0 & 0 & 0 & 1 & 0 & 0 & 0 & 0 & 0 & 0 & 0 & 0 & 0 & 0 & 0 & 0 & 0 & 0 & 0 & 0 \\
\hline K13 & 4 & 2 & 1 & 0 & 0 & 1 & 0 & 0 & 0 & 0 & 0 & 0 & 0 & 0 & 0 & 0 & 0 & 0 & 0 & 0 & 0 & 0 & 0 & 0 & 0 & 0 & 0 & 0 & 0 & 0 & 0 & 0 & 0 & 0 & 0 & 0 & 0 & 0 & 0 & 0 & 0 \\
\hline K14 & 415 & 492 & 330 & 247 & 153 & 123 & 91 & 70 & 52 & 47 & 45 & 23 & 40 & 24 & 18 & 8 & 10 & 21 & 9 & 10 & 8 & 5 & 9 & 3 & 5 & 4 & 7 & 9 & 3 & 3 & 1 & 5 & 2 & 5 & 2 & 1 & 0 & 3 & 2 & 3 & 30 \\
\hline K15 & 717 & 442 & 106 & 60 & 22 & 6 & 6 & 3 & 2 & 0 & 0 & 0 & 1 & 0 & 0 & 0 & 0 & 0 & 1 & 0 & 0 & 0 & 0 & 0 & 0 & 0 & 0 & 0 & 0 & 0 & 0 & 0 & 0 & 0 & 0 & 0 & 0 & 0 & 0 & 0 & 1 \\
\hline K16 & 872 & 502 & 148 & 56 & 28 & 12 & 0 & 0 & 4 & 2 & 0 & 2 & 0 & 0 & 0 & 0 & 0 & 0 & 0 & 0 & 0 & 0 & 0 & 0 & 0 & 0 & 0 & 0 & 0 & 0 & 0 & 0 & 0 & 0 & 0 & 0 & 0 & 0 & 0 & 0 & 0 \\
\hline K18 & 34 & 19 & 1 & 2 & 3 & 0 & 0 & 0 & 0 & 0 & 0 & 0 & 0 & 0 & 0 & 0 & 0 & 0 & 0 & 0 & 0 & 0 & 0 & 0 & 0 & 0 & 0 & 0 & 0 & 0 & 0 & 0 & 0 & 0 & 0 & 0 & 0 & 0 & 0 & 0 & 0 \\
\hline K19 & 345 & 156 & 43 & 22 & 10 & 9 & 9 & 2 & 4 & 3 & 0 & 3 & 2 & 2 & 0 & 2 & 0 & 1 & 1 & 0 & 0 & 0 & 1 & 0 & 0 & 0 & 1 & 0 & 0 & 0 & 0 & 0 & 0 & 0 & 0 & 0 & 0 & 0 & 0 & 0 & 0 \\
\hline K20 & 812 & 570 & 316 & 159 & 94 & 100 & 39 & 33 & 28 & 17 & 11 & 10 & 11 & 2 & 6 & 4 & 7 & 1 & 4 & 1 & 3 & 2 & 1 & 0 & 1 & 0 & 0 & 0 & 1 & 1 & 3 & 0 & 1 & 0 & 1 & 1 & 1 & 1 & 0 & 0 & 15 \\
\hline K21 & 53 & 16 & 5 & 3 & 1 & 2 & 0 & 0 & 0 & 1 & 0 & 0 & 0 & 0 & 0 & 0 & 0 & 0 & 0 & 0 & 0 & 0 & 0 & 0 & 0 & 0 & 1 & 0 & 0 & 0 & 0 & 0 & 0 & 0 & 0 & 0 & 0 & 0 & 0 & 0 & 0 \\
\hline K22 & 182 & 101 & 30 & 22 & 15 & 10 & 3 & 5 & 5 & 2 & 4 & 2 & 2 & 3 & 2 & 3 & 2 & 1 & 1 & 1 & 1 & 1 & 2 & 0 & 0 & 1 & 1 & 0 & 2 & 0 & 0 & 0 & 1 & 0 & 0 & 0 & 0 & 1 & 0 & 1 & 5 \\
\hline K23 & 14 & 7 & 3 & 1 & 7 & 3 & 3 & 1 & 0 & 0 & 1 & 0 & 0 & 0 & 1 & 0 & 0 & 1 & 0 & 1 & 0 & 0 & 0 & 0 & 0 & 0 & 1 & 0 & 0 & 1 & 0 & 0 & 0 & 0 & 0 & 0 & 0 & 0 & 0 & 1 & 5 \\
\hline K24 & 75 & 33 & 27 & 14 & 13 & 7 & 11 & 5 & 6 & 10 & 1 & 4 & 2 & 6 & 4 & 1 & 3 & 0 & 0 & 3 & 2 & 1 & 2 & 1 & 1 & 1 & 2 & 2 & 0 & 1 & 0 & 1 & 1 & 1 & 0 & 0 & 2 & 0 & 1 & 0 & 18 \\
\hline K25 & 2 & 2 & 3 & 1 & 2 & 0 & 0 & 0 & 0 & 0 & 0 & 0 & 0 & 0 & 0 & 0 & 0 & 0 & 0 & 0 & 0 & 0 & 0 & 0 & 0 & 0 & 0 & 0 & 0 & 0 & 0 & 0 & 0 & 0 & 0 & 0 & 0 & 0 & 0 & 0 & 0 \\
\hline K26 & 2 & 2 & 0 & 0 & 0 & 0 & 0 & 0 & 0 & 0 & 0 & 0 & 0 & 0 & 0 & 0 & 0 & 0 & 0 & 0 & 0 & 0 & 0 & 0 & 0 & 0 & 0 & 0 & 0 & 0 & 0 & 0 & 0 & 0 & 0 & 0 & 0 & 0 & 0 & 1 & 0 \\
\hline K27 & 22 & 25 & 19 & 20 & 9 & 10 & 6 & 10 & 5 & 4 & 3 & 4 & 3 & 3 & 3 & 4 & 0 & 1 & 1 & 1 & 3 & 2 & 1 & 1 & 0 & 0 & 0 & 0 & 0 & 0 & 0 & 0 & 1 & 0 & 0 & 0 & 0 & 1 & 0 & 1 & 5 \\
\hline K28 & 4 & 2 & 4 & 2 & 3 & 2 & 0 & 1 & 1 & 0 & 0 & 0 & 0 & 0 & 0 & 1 & 0 & 2 & 0 & 0 & 1 & 0 & 1 & 0 & 0 & 0 & 0 & 0 & 0 & 0 & 0 & 0 & 0 & 0 & 0 & 0 & 0 & 0 & 0 & 0 & 0 \\
\hline K29 & 130 & 189 & 168 & 123 & 103 & 67 & 66 & 40 & 32 & 29 & 25 & 34 & 26 & 17 & 23 & 18 & 15 & 6 & 8 & 7 & 8 & 4 & 6 & 6 & 4 & 6 & 2 & 7 & 6 & 4 & 5 & 4 & 8 & 7 & 3 & 2 & 2 & 2 & 5 & 3 & 132 \\
\hline K30 & 18 & 7 & 5 & 9 & 8 & 6 & 5 & 3 & 1 & 1 & 4 & 4 & 2 & 5 & 2 & 1 & 0 & 2 & 2 & 2 & 2 & 0 & 2 & 1 & 0 & 2 & 1 & 0 & 0 & 2 & 1 & 2 & 0 & 1 & 0 & 0 & 1 & 0 & 0 & 0 & 20 \\
\hline K31 & 917 & 639 & 207 & 91 & 25 & 19 & 8 & 4 & 4 & 4 & 1 & 1 & 2 & 1 & 0 & 1 & 0 & 0 & 1 & 1 & 0 & 0 & 0 & 0 & 1 & 1 & 1 & 0 & 1 & 1 & 0 & 1 & 0 & 0 & 0 & 0 & 0 & 0 & 0 & 0 & 5 \\
\hline K32 & 61 & 27 & 8 & 9 & 4 & 9 & 3 & 4 & 7 & 0 & 2 & 1 & 0 & 2 & 0 & 0 & 0 & 0 & 2 & 0 & 2 & 0 & 6 & 0 & 0 & 2 & 0 & 0 & 0 & 0 & 0 & 0 & 0 & 0 & 0 & 0 & 0 & 0 & 0 & 0 & 0 \\
\hline K33 & 454 & 135 & 52 & 23 & 11 & 13 & 4 & 2 & 0 & 0 & 3 & 0 & 0 & 0 & 0 & 2 & 0 & 4 & 2 & 0 & 1 & 0 & 0 & 0 & 0 & 0 & 0 & 2 & 0 & 0 & 0 & 0 & 0 & 0 & 0 & 0 & 0 & 0 & 0 & 0 & 0 \\
\hline
\end{tabular}


Table 5. Cont

\begin{tabular}{|c|c|c|c|c|c|c|c|c|c|c|c|c|c|c|c|c|c|c|c|c|c|c|c|c|c|c|c|c|c|c|c|c|c|c|c|c|c|c|c|c|c|}
\hline Ranges & P1 & P2 & P3 & P4 & P5 & P6 & P7 & P8 & P9 & P10 & P11 & P12 & P13 & P14 & P15 & P16 & P17 & P18 & P19 & P20 & P21 & P22 & P23 & P24 & P25 & P26 & P27 & $\begin{array}{l}\text { P28 } \\
\end{array}$ & P29 & P30 & P31 & P32 & P33 & P34 & P35 & P36 & P37 & P38 & P39 & P40 & P41 \\
\hline \multicolumn{42}{|l|}{ Category } \\
\hline K34 & 124 & 60 & 22 & 9 & 4 & 7 & 4 & 4 & 2 & 2 & 2 & 0 & 0 & 0 & 0 & 0 & 0 & 0 & 0 & 0 & 0 & 0 & 0 & 0 & 0 & 0 & 0 & 0 & 0 & 0 & 0 & 0 & 0 & 0 & 0 & 0 & 0 & 0 & 0 & 0 & 2 \\
\hline K35 & 133 & 48 & 33 & 6 & 11 & 4 & 4 & 3 & 4 & 1 & 1 & 1 & 0 & 0 & 1 & 0 & 0 & 0 & 0 & 0 & 0 & 0 & 0 & 0 & 0 & 0 & 0 & 0 & 0 & 0 & 0 & 0 & 0 & 0 & 0 & 0 & 0 & 0 & 0 & 0 & 0 \\
\hline K36 & 312 & 225 & 162 & 106 & 73 & 58 & 32 & 17 & 13 & 12 & 8 & 6 & 2 & 6 & 4 & 2 & 1 & 5 & 1 & 1 & 1 & 2 & 0 & 1 & 1 & 1 & 1 & 0 & 0 & 0 & 0 & 1 & 0 & 0 & 0 & 0 & 0 & 1 & 0 & 0 & 3 \\
\hline K37 & 237 & 87 & 30 & 18 & 9 & 8 & 7 & 7 & 3 & 6 & 2 & 1 & 2 & 0 & 0 & 5 & 1 & 2 & 0 & 0 & 2 & 1 & 0 & 0 & 0 & 0 & 1 & 0 & 0 & 0 & 0 & 0 & 0 & 0 & 1 & 0 & 0 & 1 & 0 & 0 & 3 \\
\hline K38 & 78 & 26 & 8 & 4 & 4 & 2 & 2 & 0 & 1 & 0 & 0 & 0 & 0 & 0 & 0 & 0 & 0 & 1 & 0 & 0 & 0 & 0 & 0 & 0 & 0 & 0 & 0 & 0 & 0 & 0 & 0 & 0 & 0 & 0 & 0 & 0 & 0 & 0 & 0 & 0 & 1 \\
\hline K39 & 429 & 184 & 46 & 13 & 8 & 10 & 8 & 3 & 2 & 1 & 1 & 0 & 0 & 0 & 1 & 0 & 0 & 0 & 0 & 0 & 0 & 1 & 0 & 0 & 0 & 0 & 0 & 0 & 1 & 0 & 0 & 0 & 0 & 1 & 0 & 0 & 0 & 0 & 0 & 0 & 0 \\
\hline K40 & 227 & 133 & 59 & 31 & 27 & 18 & 15 & 10 & 5 & 7 & 4 & 3 & 4 & 2 & 1 & 1 & 1 & 0 & 4 & 1 & 2 & 1 & 2 & 1 & 1 & 0 & 0 & 0 & 0 & 1 & 1 & 0 & 1 & 0 & 0 & 0 & 0 & 0 & 0 & 0 & 7 \\
\hline K42 & 79 & 67 & 57 & 36 & 41 & 33 & 32 & 15 & 15 & 20 & 15 & 14 & 14 & 8 & 11 & 9 & 15 & 5 & 10 & 7 & 7 & 4 & 2 & 1 & 5 & 1 & 3 & 2 & 1 & 4 & 5 & 3 & 3 & 0 & 3 & 0 & 2 & 4 & 4 & 3 & 66 \\
\hline
\end{tabular}

Table 6. Numerical input data in terms of campaign duration (categories/ranges).

\begin{tabular}{|c|c|c|c|c|c|c|c|c|c|c|c|c|c|c|c|c|c|c|c|c|c|c|c|c|c|c|c|c|c|c|c|}
\hline Ranges. & P1 & P2 & P3 & P4 & P5 & P6 & P7 & P8 & P9 & P10 & P11 & P12 & P13 & P14 & P15 & P16 & P17 & P18 & P19 & P20 & P21 & P22 & P23 & P24 & P25 & P26 & P27 & P28 & P29 & P30 & P31 \\
\hline \multicolumn{32}{|l|}{ Category } \\
\hline K1 & 0 & 0 & 4 & 18 & 6 & 11 & 54 & 73 & 33 & 75 & 74 & 32 & 52 & 27 & 321 & 80 & 5 & 18 & 5 & 6 & 4 & 4 & 9 & 2 & 3 & 2 & 1 & 1 & 0 & 12 & 6 \\
\hline K2 & 0 & 1 & 0 & 1 & 0 & 1 & 9 & 2 & 5 & 5 & 10 & 9 & 17 & 11 & 103 & 35 & 15 & 8 & 0 & 3 & 0 & 0 & 6 & 0 & 1 & 0 & 1 & 0 & 0 & 13 & 0 \\
\hline K4 & 0 & 0 & 0 & 1 & 1 & 3 & 5 & 8 & 4 & 11 & 11 & 2 & 5 & 6 & 54 & 17 & 3 & 6 & 4 & 1 & 1 & 0 & 2 & 0 & 1 & 3 & 0 & 0 & 0 & 2 & 1 \\
\hline K5 & 0 & 1 & 1 & 7 & 3 & 8 & 20 & 51 & 16 & 36 & 47 & 26 & 33 & 30 & 224 & 73 & 14 & 17 & 4 & 6 & 6 & 3 & 8 & 1 & 2 & 2 & 0 & 4 & 0 & 15 & 0 \\
\hline K6 & 0 & 0 & 0 & 0 & 0 & 0 & 0 & 1 & 1 & 0 & 0 & 0 & 0 & 0 & 2 & 1 & 0 & 0 & 0 & 0 & 0 & 0 & 1 & 0 & 1 & 0 & 0 & 0 & 0 & 0 & 0 \\
\hline K7 & 0 & 0 & 0 & 0 & 2 & 2 & 5 & 4 & 5 & 4 & 6 & 2 & 5 & 4 & 24 & 11 & 2 & 2 & 2 & 3 & 0 & 3 & 2 & 0 & 0 & 0 & 0 & 0 & 0 & 4 & 0 \\
\hline K9 & 0 & 0 & 0 & 1 & 0 & 0 & 0 & 0 & 1 & 0 & 1 & 0 & 0 & 1 & 5 & 0 & 0 & 0 & 1 & 0 & 1 & 1 & 0 & 0 & 0 & 0 & 0 & 0 & 0 & 2 & 0 \\
\hline K10 & 0 & 0 & 0 & 0 & 0 & 0 & 1 & 0 & 0 & 6 & 2 & 3 & 1 & 1 & 13 & 2 & 0 & 1 & 0 & 1 & 0 & 0 & 0 & 0 & 0 & 1 & 0 & 0 & 0 & 1 & 0 \\
\hline K11 & 0 & 0 & 0 & 0 & 0 & 0 & 1 & 1 & 0 & 0 & 1 & 0 & 0 & 1 & 7 & 3 & 2 & 0 & 1 & 1 & 0 & 1 & 0 & 0 & 0 & 0 & 0 & 0 & 0 & 0 & 1 \\
\hline K12 & 0 & 0 & 0 & 0 & 0 & 0 & 0 & 1 & 0 & 2 & 1 & 1 & 0 & 0 & 9 & 3 & 1 & 2 & 1 & 0 & 0 & 0 & 0 & 0 & 0 & 0 & 1 & 0 & 0 & 1 & 0 \\
\hline K13 & 0 & 0 & 0 & 0 & 0 & 0 & 0 & 0 & 0 & 0 & 0 & 0 & 1 & 0 & 4 & 0 & 0 & 0 & 0 & 0 & 0 & 0 & 1 & 1 & 0 & 0 & 0 & 0 & 0 & 1 & 0 \\
\hline K14 & 0 & 3 & 2 & 6 & 17 & 14 & 27 & 58 & 19 & 38 & 73 & 51 & 65 & 51 & 1041 & 389 & 104 & 91 & 47 & 44 & 17 & 20 & 46 & 4 & 7 & 5 & 4 & 3 & 4 & 68 & 20 \\
\hline K15 & 255 & 1 & 5 & 8 & 10 & 8 & 20 & 32 & 15 & 52 & 62 & 27 & 56 & 52 & 348 & 118 & 44 & 25 & 27 & 39 & 16 & 24 & 35 & 9 & 8 & 6 & 6 & 7 & 7 & 42 & 3 \\
\hline K16 & 334 & 2 & 1 & 10 & 13 & 11 & 14 & 36 & 21 & 35 & 76 & 40 & 69 & 55 & 403 & 120 & 63 & 69 & 33 & 34 & 13 & 13 & 34 & 7 & 10 & 12 & 4 & 3 & 6 & 72 & 13 \\
\hline K18 & 11 & 1 & 0 & 0 & 0 & 0 & 1 & 1 & 2 & 1 & 2 & 0 & 2 & 3 & 13 & 10 & 2 & 0 & 0 & 1 & 0 & 0 & 2 & 0 & 1 & 0 & 0 & 0 & 1 & 5 & 0 \\
\hline
\end{tabular}


Table 6. Cont.

\begin{tabular}{|c|c|c|c|c|c|c|c|c|c|c|c|c|c|c|c|c|c|c|c|c|c|c|c|c|c|c|c|c|c|c|c|}
\hline Ranges. & P1 & P2 & P3 & P4 & P5 & P6 & P7 & P8 & P9 & P10 & P11 & P12 & P13 & P14 & P15 & P16 & P17 & P18 & P19 & $\mathbf{P 2 0}$ & P21 & $\mathbf{P 2 2}$ & P23 & P24 & P25 & P26 & P27 & P28 & P29 & P30 & P31 \\
\hline \multicolumn{32}{|l|}{ Category } \\
\hline K19 & 109 & 2 & 4 & 6 & 7 & 3 & 17 & 25 & 8 & 18 & 31 & 12 & 19 & 14 & 215 & 53 & 11 & 25 & 1 & 8 & 1 & 2 & 11 & 1 & 2 & 0 & 0 & 0 & 0 & 9 & 2 \\
\hline K20 & 414 & 1 & 1 & 7 & 15 & 8 & 18 & 38 & 17 & 44 & 73 & 30 & 68 & 52 & 677 & 182 & 73 & 84 & 37 & 57 & 29 & 20 & 83 & 17 & 14 & 20 & 7 & 8 & 9 & 92 & 62 \\
\hline K21 & 15 & 0 & 0 & 1 & 1 & 0 & 2 & 6 & 1 & 7 & 2 & 1 & 1 & 3 & 21 & 7 & 1 & 3 & 0 & 2 & 1 & 0 & 0 & 1 & 1 & 1 & 0 & 0 & 0 & 3 & 1 \\
\hline K22 & 84 & 1 & 1 & 4 & 2 & 3 & 12 & 7 & 5 & 15 & 7 & 11 & 12 & 13 & 115 & 34 & 14 & 12 & 3 & 11 & 5 & 3 & 11 & 2 & 0 & 1 & 2 & 2 & 2 & 17 & 1 \\
\hline K23 & 8 & 0 & 1 & 0 & 0 & 2 & 6 & 4 & 1 & 1 & 2 & 2 & 0 & 2 & 15 & 2 & 1 & 0 & 0 & 3 & 0 & 0 & 0 & 0 & 0 & 0 & 0 & 0 & 0 & 1 & 0 \\
\hline K24 & 56 & 0 & 0 & 0 & 1 & 1 & 2 & 5 & 0 & 5 & 7 & 5 & 4 & 4 & 92 & 26 & 6 & 9 & 4 & 9 & 3 & 3 & 5 & 2 & 1 & 0 & 0 & 0 & 0 & 11 & 1 \\
\hline K25 & 0 & 0 & 0 & 0 & 0 & 0 & 1 & 0 & 0 & 0 & 0 & 0 & 0 & 0 & 4 & 2 & 0 & 1 & 1 & 0 & 0 & 0 & 0 & 0 & 0 & 0 & 0 & 0 & 0 & 1 & 0 \\
\hline K26 & 2 & 0 & 1 & 0 & 0 & 0 & 0 & 0 & 0 & 0 & 0 & 0 & 0 & 0 & 1 & 0 & 0 & 1 & 0 & 0 & 0 & 0 & 0 & 0 & 0 & 0 & 0 & 0 & 0 & 0 & 0 \\
\hline K27 & 33 & 0 & 0 & 3 & 1 & 2 & 5 & 6 & 5 & 3 & 6 & 5 & 5 & 7 & 58 & 11 & 1 & 1 & 5 & 2 & 2 & 0 & 1 & 0 & 3 & 0 & 0 & 0 & 0 & 3 & 0 \\
\hline K28 & 3 & 0 & 0 & 1 & 0 & 0 & 0 & 2 & 0 & 1 & 1 & 1 & 2 & 1 & 10 & 1 & 0 & 1 & 0 & 0 & 0 & 0 & 0 & 0 & 0 & 0 & 0 & 0 & 0 & 0 & 0 \\
\hline K29 & 280 & 2 & 5 & 22 & 27 & 17 & 199 & 94 & 50 & 40 & 86 & 42 & 45 & 35 & 289 & 65 & 6 & 13 & 2 & 8 & 1 & 3 & 7 & 1 & 1 & 0 & 0 & 0 & 0 & 12 & 0 \\
\hline K30 & 28 & 0 & 0 & 0 & 0 & 0 & 1 & 2 & 0 & 0 & 1 & 1 & 4 & 2 & 54 & 13 & 1 & 3 & 3 & 0 & 1 & 0 & 3 & 0 & 0 & 0 & 0 & 0 & 0 & 5 & 0 \\
\hline K31 & 211 & 1 & 2 & 12 & 14 & 14 & 22 & 26 & 33 & 36 & 94 & 33 & 49 & 48 & 589 & 163 & 52 & 68 & 37 & 57 & 33 & 22 & 65 & 16 & 14 & 15 & 13 & 9 & 8 & 138 & 43 \\
\hline K32 & 17 & 0 & 0 & 2 & 2 & 0 & 8 & 5 & 0 & 2 & 8 & 6 & 10 & 7 & 42 & 14 & 1 & 6 & 0 & 5 & 3 & 1 & 0 & 0 & 0 & 0 & 0 & 0 & 0 & 8 & 2 \\
\hline K33 & 59 & 0 & 33 & 66 & 11 & 15 & 6 & 20 & 5 & 19 & 34 & 2 & 21 & 17 & 213 & 75 & 18 & 16 & 8 & 4 & 10 & 6 & 18 & 0 & 1 & 2 & 4 & 2 & 2 & 20 & 1 \\
\hline K34 & 34 & 0 & 0 & 3 & 3 & 2 & 5 & 4 & 5 & 5 & 7 & 5 & 8 & 9 & 75 & 31 & 4 & 3 & 5 & 5 & 1 & 2 & 11 & 0 & 0 & 2 & 0 & 0 & 0 & 5 & 8 \\
\hline K35 & 32 & 1 & 0 & 3 & 5 & 4 & 4 & 7 & 6 & 7 & 5 & 6 & 7 & 4 & 81 & 20 & 6 & 7 & 1 & 4 & 1 & 1 & 11 & 1 & 1 & 2 & 1 & 0 & 1 & 16 & 5 \\
\hline K36 & 110 & 2 & 20 & 16 & 9 & 5 & 3 & 17 & 11 & 15 & 51 & 19 & 22 & 34 & 387 & 111 & 36 & 40 & 20 & 20 & 6 & 7 & 31 & 7 & 6 & 3 & 3 & 1 & 1 & 38 & 7 \\
\hline K37 & 46 & 0 & 2 & 1 & 6 & 5 & 7 & 12 & 5 & 16 & 14 & 10 & 13 & 15 & 144 & 28 & 14 & 12 & 4 & 15 & 5 & 4 & 15 & 6 & 3 & 4 & 0 & 0 & 3 & 23 & 2 \\
\hline K38 & 16 & 0 & 0 & 2 & 2 & 2 & 1 & 6 & 1 & 6 & 1 & 5 & 2 & 4 & 43 & 11 & 5 & 4 & 0 & 1 & 1 & 0 & 3 & 1 & 3 & 0 & 0 & 1 & 0 & 5 & 1 \\
\hline K39 & 87 & 2 & 4 & 7 & 3 & 3 & 12 & 19 & 7 & 17 & 32 & 10 & 14 & 15 & 257 & 53 & 21 & 28 & 11 & 14 & 9 & 6 & 20 & 0 & 4 & 6 & 0 & 6 & 1 & 28 & 13 \\
\hline K40 & 67 & 0 & 3 & 4 & 2 & 5 & 10 & 10 & 5 & 12 & 22 & 6 & 16 & 9 & 211 & 50 & 18 & 13 & 3 & 10 & 11 & 8 & 20 & 1 & 2 & 5 & 3 & 2 & 2 & 33 & 7 \\
\hline K41 & 6 & 0 & 0 & 0 & 0 & 0 & 0 & 1 & 1 & 0 & 1 & 1 & 0 & 0 & 17 & 4 & 4 & 1 & 2 & 2 & 0 & 0 & 3 & 1 & 1 & 0 & 0 & 0 & 0 & 0 & 4 \\
\hline K42 & 0 & 0 & 0 & 0 & 0 & 0 & 0 & 0 & 0 & 0 & 0 & 0 & 0 & 0 & 0 & 0 & 0 & 0 & 0 & 0 & 0 & 0 & 0 & 0 & 0 & 0 & 0 & 0 & 0 & 0 & 8 \\
\hline
\end{tabular}


Table 7. Numerical input data in terms of project profitability (categories/ranges).

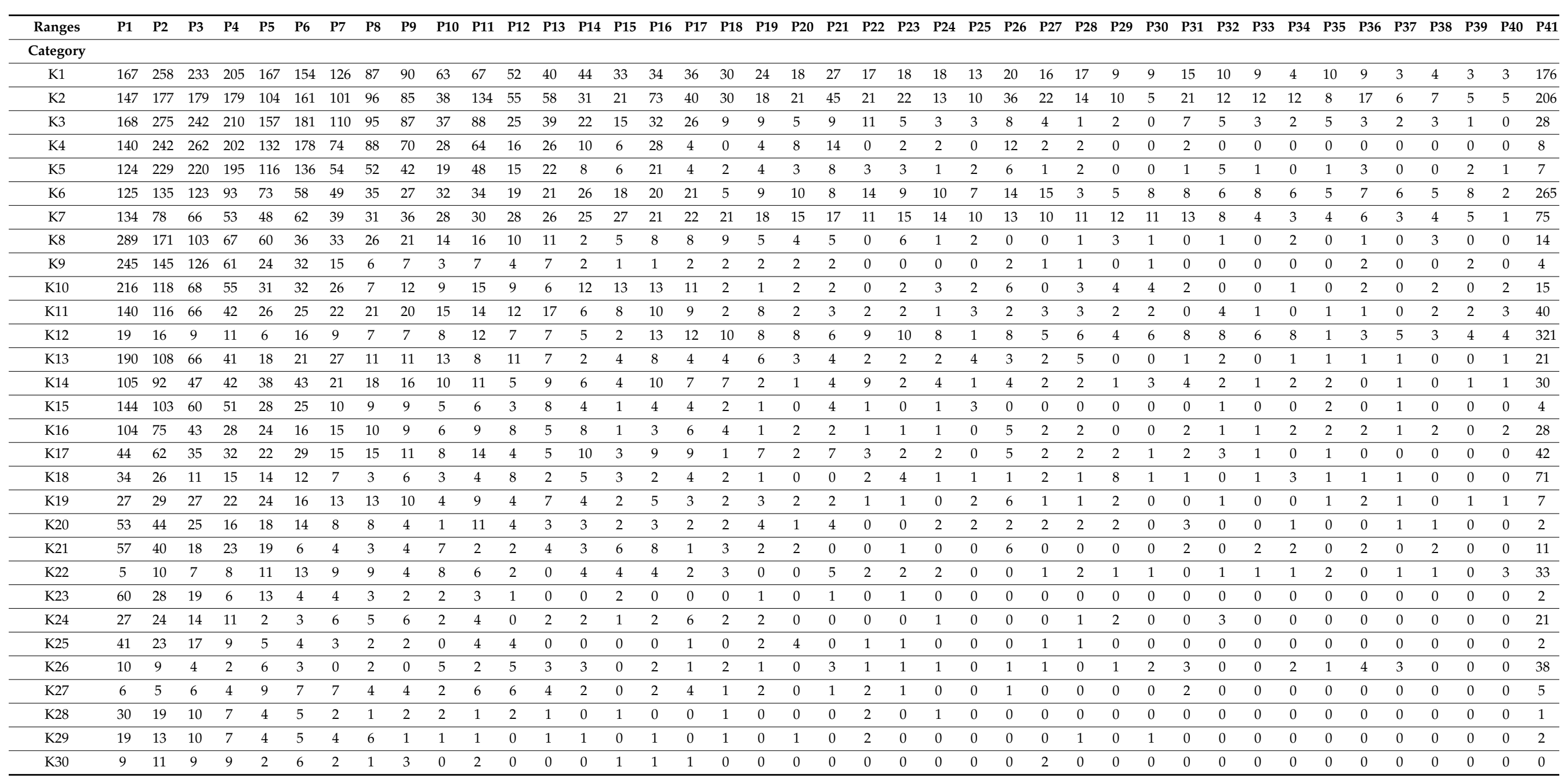


Table 7. Cont.

\begin{tabular}{|c|c|c|c|c|c|c|c|c|c|c|c|c|c|c|c|c|c|c|c|c|c|c|c|c|c|c|c|c|c|c|c|c|c|c|c|c|c|c|c|c|c|}
\hline Ranges & P1 & P2 & P3 & P4 & P5 & P6 & P7 & P8 & P9 & P10 & P11 & P12 & P13 & P14 & P15 & P16 & P17 & P18 & P19 & $\mathbf{P 2 0}$ & $\mathbf{P 2 1}$ & P22 & P23 & P24 & P25 & P26 & P27 & P28 & P29 & P30 & P31 & P32 & P33 & P34 & P35 & P36 & P37 & P38 & P39 & P40 & P41 \\
\hline \multicolumn{42}{|l|}{ Category } \\
\hline K31 & 12 & 10 & 4 & 5 & 3 & 3 & 3 & 2 & 1 & 0 & 2 & 0 & 0 & 0 & 0 & 0 & 0 & 1 & 0 & 2 & 0 & 0 & 1 & 0 & 0 & 0 & 1 & 0 & 0 & 0 & 0 & 0 & 0 & 0 & 0 & 0 & 0 & 0 & 0 & 0 & 4 \\
\hline K32 & 6 & 7 & 1 & 2 & 6 & 2 & 1 & 1 & 0 & 0 & 2 & 1 & 0 & 3 & 1 & 2 & 2 & 1 & 0 & 1 & 1 & 0 & 0 & 0 & 0 & 0 & 0 & 0 & 0 & 0 & 0 & 0 & 0 & 0 & 0 & 0 & 0 & 0 & 0 & 0 & 11 \\
\hline K34 & 4 & 4 & 2 & 5 & 4 & 1 & 2 & 2 & 2 & 0 & 0 & 0 & 0 & 1 & 1 & 1 & 0 & 0 & 1 & 0 & 0 & 0 & 0 & 0 & 0 & 0 & 0 & 0 & 0 & 0 & 0 & 0 & 0 & 1 & 0 & 0 & 0 & 0 & 0 & 0 & 2 \\
\hline K35 & 0 & 3 & 0 & 1 & 2 & 2 & 1 & 1 & 2 & 0 & 0 & 1 & 1 & 0 & 0 & 1 & 2 & 0 & 0 & 0 & 0 & 1 & 1 & 1 & 0 & 0 & 0 & 0 & 0 & 0 & 0 & 0 & 0 & 0 & 0 & 0 & 0 & 0 & 0 & 0 & 4 \\
\hline K36 & 3 & 3 & 2 & 0 & 0 & 3 & 2 & 1 & 0 & 1 & 0 & 2 & 1 & 2 & 0 & 0 & 1 & 0 & 0 & 1 & 0 & 0 & 0 & 0 & 0 & 0 & 0 & 0 & 0 & 0 & 0 & 0 & 0 & 0 & 0 & 0 & 0 & 0 & 0 & 0 & 1 \\
\hline K37 & 5 & 3 & 2 & 3 & 0 & 1 & 1 & 1 & 0 & 0 & 0 & 0 & 0 & 0 & 0 & 0 & 0 & 0 & 1 & 0 & 1 & 0 & 0 & 0 & 0 & 0 & 0 & 1 & 0 & 0 & 0 & 0 & 0 & 0 & 0 & 0 & 0 & 0 & 0 & 0 & 1 \\
\hline K39 & 0 & 2 & 0 & 0 & 1 & 1 & 0 & 1 & 1 & 0 & 0 & 0 & 1 & 1 & 0 & 0 & 0 & 0 & 0 & 0 & 0 & 0 & 1 & 0 & 0 & 0 & 0 & 0 & 0 & 0 & 0 & 0 & 1 & 0 & 0 & 0 & 0 & 0 & 0 & 0 & 0 \\
\hline K40 & 1 & 2 & 1 & 0 & 0 & 1 & 0 & 0 & 0 & 0 & 1 & 0 & 0 & 0 & 0 & 0 & 0 & 1 & 0 & 0 & 0 & 0 & 0 & 0 & 0 & 0 & 0 & 1 & 0 & 0 & 0 & 0 & 0 & 0 & 0 & 0 & 0 & 0 & 0 & 0 & 0 \\
\hline K41 & 1 & 1 & 0 & 1 & 0 & 0 & 0 & 0 & 0 & 1 & 0 & 0 & 0 & 0 & 0 & 0 & 1 & 0 & 0 & 0 & 0 & 0 & 0 & 0 & 0 & 0 & 0 & 0 & 0 & 0 & 0 & 0 & 0 & 0 & 0 & 1 & 0 & 0 & 0 & 0 & 1 \\
\hline K42 & 2 & 0 & 0 & 0 & 0 & 0 & 1 & 0 & 0 & 0 & 0 & 0 & 0 & 0 & 0 & 1 & 0 & 0 & 0 & 0 & 0 & 0 & 0 & 0 & 0 & 0 & 0 & 0 & 0 & 0 & 0 & 0 & 0 & 0 & 0 & 0 & 0 & 0 & 0 & 0 & 1 \\
\hline
\end{tabular}

Source: authors' own compilation based on webrobots.io/kickstarter-datasets, data from 17 June 2021. 
The second concept, also known as the concept of linguistic classification, presents a more traditional approach to this issue. This approach is represented by Ishibuchi et al. [69], who believe that classifying is an act of dividing a set into classes. The result of this activity is a classification, i.e., a set of features with specific relations between their ranges and the classified set.

One of the basic logical requirements of correct classification is the postulate of being complete and disjoint. Being complete requires that each element belonging to the classified set must belong to at least one group (class), whereas being disjoint means that the scopes of individual classes should not overlap. Both postulates of logical correctness of classification require that each element within the scope of the classified set must belong to one and only one class, and therefore that neither element is omitted while classifying nor classified more than once.

Classification should also be rational. Rationality ensures the optimal implementation of the purpose for which this classification was created. The rationality of classification is subject to relativization with regard to the classified set and the purpose of the classification. A desirable feature of classification is also its clarity, i.e., how easy it is to navigate its structure. The use of classification as a tool for understanding reality improves the orientation in the apparent chaos of objects.

The academic literature $[70,71]$ uses the terms "classification" and "component binding" interchangeably. The latter term is used especially when choosing the method of combining objects. There is, for example, multi-aspect binding (according to many criteria) and single-aspect binding (according to one criterion).

The so-called royalty, that is a measure of the closeness centrality (similarity) of elements, is usually assumed as the basis of classification. It should be noted that the selection of the similarity function does not significantly affect the result of binding. The measure of similarity has been successfully used by many authors of methods for classification and information retrieval. Some of these methods require determining a priori the target number of classes, e.g., factor analysis [72]. Other more classical methods, for instance, the Rocchio [73] algorithm or Hill [74] method, allow us to regulate the number of classes. The Vaswani method [75] uses indirect connections between the elements of future classes, and the method developed by Datolii $[76,77]$ assumes that the set is a priori divided into socalled starting classes. Some methods are based on single-aspect classification, and others use more criteria, e.g., the Gitman-Lewine method [78]. This is the approach adopted in this article.

The criterion for qualitative evaluation of a particular binding algorithm is complex, subjective and dependent on predetermined parameters. Such parameters may include the number of elements, their coherence, the degree of internal homogeneity, etc. Unless an unequivocal division of the set into classes is given a priori by experts, each criterion for assessing the quality of the classification method is subjective. So far, many different criteria for optimizing classification methods have been developed, for example minimizing the entropy of a set or minimizing the risk function. However, these criteria are of little use in practice. Therefore, one often has to be content with the so-called quasi-optimal classification, the assessment of which is presented in a simplified manner.

It is important to emphasize that the type of classified objects does not affect the choice of method. What does, however, influence it is the required degree of accuracy, i.e., the number of classification criteria it takes into account and whether the order of the classified objects affects the final result.

For the purposes of further discussion, it is assumed that the name is a constant, nonmeasurable component of the object that makes up the terminology system. It identifies a given object in a set of similar objects in the convention of the adopted description language. It has a scheduling (from the point of view of its form), pragmatic (from the point of view of its use in information and decision-making processes) and semantic (from the point of view of the content of the name) meaning. Value, on the other hand, is a variable and 
measurable part of the component, reflecting, for example, a measurement. Therefore, values can be considered, as already mentioned, as object occurrences.

Since solving the issue of data-identification methods is not the purpose of this paper, the identified list of data names (categories and attributes) was taken as a starting point for further considerations. The binding method should therefore meet the following conditions:

- It cannot depend on a set of bound components,

- The order in which the objects are bound would not affect the result of binding,

- The result of binding must be stable in the sense that a small change in the set of objects will also cause small changes in the result,

- Objects similar to each other must belong to the same class,

- The amount of average loss resulting from an incorrect assignment of an object to a given class should be minimal.

The analysis of binding methods leads to the conclusion that the method of binding derived from the designer and two-aspect approach will be the closest to the assumptions adopted in the paper. The designer aspect creates the possibility to bind components omitting the limitations resulting from the actual state of the tested system. The twoaspect feature allows us to include two key criteria of binding components: closeness and validity (weight).

Taking into account the above considerations, the presented methodology can be divided into the following stages:

1. Classification of project categories $(\mathrm{K})$ according to the adopted attributes:

- Presenting data from Tables $5-7$ in the form of a $(0,1)$ matrix KA (that is, category attribute), which will have the following structure:

$$
\mathrm{KA}=\left[\begin{array}{ccc}
\mathrm{p}_{11} & \cdots & \mathrm{p}_{1 \mathrm{j}} \\
\vdots & \ddots & \vdots \\
\mathrm{p}_{\mathrm{i} 1} & \cdots & \mathrm{p}_{\mathrm{ij}}
\end{array}\right]
$$

where $\mathrm{p}_{\mathrm{ij}}=\{1$-when the category contains projects related to a given attribute within the specified range, 0 - otherwise

- Transforming the KA matrix into the area of category proximity, also presented in the form of the matrix: BKA. The following proximity function was adopted as the basis for the transformation:

$$
\mathrm{b}\left(\mathrm{k}_{\mathrm{i}}, \mathrm{k}_{\mathrm{j}}\right)=\frac{\mathrm{p}_{\mathrm{i}} \cap \mathrm{p}_{\mathrm{j}}}{\mathrm{p}_{\mathrm{i}} \cup \mathrm{p}_{\mathrm{j}}}, \text { where } \mathrm{b} \in<0,1>,
$$

- Based on this function, the pairs of categories from the KA area are compared and the logical conjunction and the logical disjunction equal to 1 are determined. The rows and columns of the BKA matrix correspond to the categories, and its elements are the values of the proximity function, the so-called closeness factors:

$$
\text { BKA }=\left[\begin{array}{ccc}
b_{11} & \cdots & b_{1 j} \\
\vdots & \ddots & \vdots \\
b_{i 1} & \cdots & b_{i j}
\end{array}\right], \text { wherein, for } i=j, b i j=1 \text { is adopted, }
$$

It is worth emphasizing that the presented procedure does not use the conversion of the BKA matrix to a binary matrix, as in other methods, because this conversion, based on the assumption of a certain subjective threshold value, would reduce the accuracy of the formula.

2. Creating chains of $K_{w}$ and $K_{b}$ categories: the $K_{w}$ chain consists of categories ordered by weight, and the $\mathrm{K}_{\mathrm{b}}$ chain contains categories ordered by decreasing values of the 
$b$ function (diminishing similarities), i.e., $k_{i}$ precedes $k_{j}$ if $b\left(k_{1 b}, k_{i b}\right)>b\left(k_{1 b}, k_{j b}\right)$. If $b\left(k_{1 b}, k_{i b}\right)=b\left(k_{1 b}, k_{j b}\right)$, and then the $k_{i b}$ category precedes the $k_{j b}$ category if its weight $\mathrm{w}\left(\mathrm{k}_{\mathrm{ib}}\right)>\mathrm{w}\left(\mathrm{k}_{\mathrm{jb}}\right)$. If, however, $\mathrm{w}\left(\mathrm{k}_{\mathrm{ib}}\right)=\mathrm{w}\left(\mathrm{k}_{\mathrm{jb}}\right)$, then the order of the categories in the chain is arbitrary;

3. Comparing the components of so formed chains $\mathrm{K}_{\mathrm{w}}$ and $\mathrm{K}_{\mathrm{b}}$, i.e., weight and category closeness chains in order to determine the minimum value of the mk parameter, i.e., the first category with a different i-th index in both chains. Tandems of previously equal categories will initially create a subset of the set of categories-the first class,

4. Cyclical repetition (until all the categories in the $\mathrm{K}_{\mathrm{w}}$ and $\mathrm{K}_{\mathrm{b}}$ chains are exhausted) of the following sequence of operations: categories with the mk index are selected from the $\mathrm{K}_{\mathrm{w}}$ chain, that is, components with the lower index in the unequal tandem, and it is then checked whether the following is true:

- This category is a candidate for more than one of the classes created so far; if so, it will be included in only one, namely in the class in which there is the category with a higher weight in terms of function $b$;

- This category is not a candidate for any of the previously created classes; then it becomes the creator of a new class; afterwards, all the single-component classes are combined into one atypical class.

As a result of carrying out the classification procedures according to the three attributes, the following three groups of classes were obtained: KLP-class of project categories according to the number of backers, KLT—class of project categories according to the campaign duration, KLF-class of project categories according to profitability.

5. After carrying out the procedure three times, three sets of classes of categories similar in terms of the examined attributes are subjected successively (i.e., class by class), to the procedure of examining their similarity on a cross-basis, i.e., all sets of classes with each other. Then the strength of correlations between the attributes is determined, using the sample table (Table 8):

Table 8. Cross-arrangement of classes of the similarity of attributes: project profitability/campaign duration.

\begin{tabular}{cccc}
\hline & KLT1 & $\ldots$ & KLTn \\
\hline KLF1 & - & - & - \\
\hline$\ldots$ & - & - & - \\
\hline KLFn & - & - & - \\
\hline
\end{tabular}

Source: authors' own study.

The presented research methodology at the category level can also be used for analyzing each category separately, for the classification of projects submitted to it. This way more detailed information is obtained. Nevertheless, it should be emphasized that project categories are more stable within one given crowdfunding platform, hence their classifications and the analysis of attributes allow for greater generalizations when formulating conclusions.

\section{Results}

After applying the methodology described above and using the prepared source data, the following results were obtained (Tables 9-11). 
Table 9. KA matrix: project categories according to the number of people backing the projects.

\begin{tabular}{|c|c|c|c|c|c|c|c|c|c|c|c|c|c|c|c|c|c|c|c|c|c|c|c|c|c|c|c|c|c|c|c|c|c|c|c|c|c|c|c|c|c|}
\hline Ranges & P1 & P2 & P3 & P4 & P5 & P6 & P7 & P8 & P9 & P10 & P11 & P12 & P13 & P14 & P15 & P16 & P17 & P18 & P19 & P20 & P21 & P22 & P23 & P24 & P25 & P26 & P27 & P28 & P29 & P30 & P31 & P32 & P33 & P34 & P35 & P36 & P37 & P38 & P39 & P40 & P41 \\
\hline \multicolumn{42}{|l|}{ Category } \\
\hline K1 & $x$ & $x$ & $x$ & $x$ & $x$ & $x$ & $x$ & X & X & $x$ & X & $x$ & $x$ & $x$ & $x$ & X & $x$ & $x$ & X & $x$ & $x$ & $x$ & $x$ & $x$ & X & X & X & X & $x$ & X & $x$ & X & $x$ & $x$ & X & X & 0 & $x$ & $x$ & $x$ & $x$ \\
\hline K2 & $\mathrm{X}$ & $X$ & $x$ & $x$ & $x$ & $x$ & $x$ & $x$ & $X$ & $x$ & $X$ & $x$ & $\mathrm{X}$ & $X$ & $x$ & $X$ & $X$ & $x$ & $X$ & $x$ & $x$ & $X$ & $X$ & 0 & $X$ & 0 & 0 & 0 & $x$ & $X$ & $X$ & 0 & $x$ & 0 & $X$ & $\mathrm{X}$ & X & $X$ & 0 & 0 & $x$ \\
\hline K3 & $\mathrm{x}$ & $\mathrm{X}$ & $x$ & $\mathrm{X}$ & $x$ & $x$ & $x$ & $x$ & $\mathrm{X}$ & $x$ & $\mathrm{X}$ & $\mathrm{X}$ & $\mathrm{x}$ & X & 0 & X & 0 & 0 & $x$ & $\mathrm{X}$ & 0 & 0 & 0 & 0 & $x$ & $x$ & $\bar{X}$ & 0 & $x$ & X & 0 & $\mathrm{X}$ & 0 & 0 & 0 & 0 & 0 & 0 & 0 & 0 & $\mathrm{X}$ \\
\hline K4 & $x$ & $\mathrm{x}$ & $x$ & $x$ & $x$ & $x$ & 0 & 0 & $x$ & $x$ & 0 & X & 0 & 0 & 0 & 0 & 0 & 0 & 0 & 0 & 0 & 0 & 0 & 0 & 0 & 0 & 0 & 0 & 0 & 0 & 0 & 0 & 0 & 0 & 0 & 0 & 0 & 0 & 0 & 0 & 0 \\
\hline K5 & $\mathrm{x}$ & $X$ & $\mathrm{x}$ & $\mathrm{X}$ & $X$ & $\mathrm{X}$ & $x$ & $x$ & $X$ & 0 & 0 & 0 & $x$ & 0 & 0 & 0 & 0 & 0 & $x$ & 0 & 0 & 0 & 0 & 0 & 0 & 0 & 0 & 0 & 0 & 0 & 0 & 0 & 0 & 0 & 0 & 0 & 0 & 0 & 0 & 0 & $x$ \\
\hline K6 & $X$ & $X$ & $x$ & $\mathrm{X}$ & $X$ & $x$ & $X$ & $\mathrm{X}$ & $X$ & $x$ & X & $x$ & $x$ & X & X & X & X & $x$ & $\mathrm{X}$ & $x$ & $x$ & $x$ & X & $x$ & $x$ & $x$ & $\mathrm{X}$ & $x$ & $x$ & X & $x$ & X & $x$ & $x$ & $x$ & $\mathrm{X}$ & X & X & X & $x$ & $x$ \\
\hline K7 & $\mathrm{X}$ & $X$ & $x$ & $x$ & $x$ & $\mathrm{X}$ & $X$ & $x$ & $X$ & $x$ & $X$ & $x$ & $\mathrm{X}$ & $X$ & $x$ & $X$ & $x$ & $X$ & $X$ & $\mathrm{X}$ & $x$ & $x$ & 0 & $X$ & $X$ & $X$ & $X$ & 0 & 0 & 0 & 0 & $X$ & 0 & 0 & 0 & 0 & 0 & $X$ & 0 & 0 & $X$ \\
\hline K8 & $\mathrm{X}$ & $\mathrm{X}$ & $\mathrm{X}$ & $\mathrm{X}$ & $X$ & $x$ & $X$ & $x$ & $x$ & $x$ & $X$ & $x$ & $x$ & 0 & $X$ & $X$ & $X$ & 0 & $X$ & 0 & 0 & 0 & 0 & 0 & $X$ & 0 & 0 & 0 & 0 & 0 & 0 & 0 & 0 & 0 & 0 & $X$ & 0 & 0 & 0 & 0 & 0 \\
\hline K10 & $x$ & $\mathrm{X}$ & $x$ & $x$ & $X$ & $x$ & $x$ & $x$ & 0 & 0 & $x$ & 0 & 0 & 0 & 0 & $x$ & 0 & $x$ & $X$ & 0 & $x$ & 0 & 0 & 0 & 0 & 0 & 0 & $x$ & 0 & 0 & 0 & 0 & 0 & 0 & 0 & 0 & 0 & 0 & 0 & 0 & 0 \\
\hline K11 & $X$ & $X$ & $x$ & $X$ & $X$ & $X$ & $X$ & $X$ & $x$ & $x$ & $X$ & $x$ & $x$ & $x$ & $x$ & 0 & $x$ & $X$ & $X$ & $x$ & $X$ & $x$ & 0 & 0 & 0 & $x$ & $\bar{X}$ & 0 & 0 & $x$ & 0 & 0 & $x$ & $x$ & 0 & $X$ & 0 & 0 & $x$ & $x$ & $x$ \\
\hline K12 & $x$ & $X$ & $x$ & $x$ & $X$ & $x$ & $x$ & $X$ & $x$ & $x$ & $x$ & $x$ & $x$ & $x$ & $x$ & $x$ & $x$ & $X$ & $X$ & $X$ & $X$ & $X$ & $x$ & $x$ & $x$ & $X$ & $X$ & $x$ & $x$ & $X$ & $x$ & $x$ & $X$ & 0 & $x$ & 0 & $x$ & $x$ & $X$ & $X$ & $X$ \\
\hline K13 & $x$ & $x$ & $x$ & $\mathrm{X}$ & $X$ & $x$ & $X$ & $X$ & $X$ & $x$ & 0 & $\mathrm{X}$ & $x$ & $X$ & 0 & $X$ & 0 & $x$ & $X$ & 0 & 0 & 0 & $X$ & 0 & 0 & 0 & $\mathrm{X}$ & 0 & 0 & 0 & 0 & 0 & 0 & 0 & 0 & 0 & 0 & 0 & 0 & 0 & 0 \\
\hline K14 & $x$ & $\mathrm{X}$ & $x$ & $x$ & $X$ & $X$ & $X$ & $X$ & $x$ & $x$ & $X$ & $X$ & $x$ & $x$ & $x$ & $x$ & $x$ & 0 & $x$ & $x$ & $x$ & $x$ & $X$ & $x$ & $x$ & 0 & 0 & 0 & 0 & $X$ & $x$ & 0 & $x$ & 0 & 0 & 0 & 0 & 0 & 0 & 0 & $x$ \\
\hline K15 & $X$ & $X$ & $x$ & $X$ & $X$ & $X$ & $X$ & $X$ & $X$ & $x$ & 0 & $X$ & $x$ & 0 & 0 & 0 & 0 & 0 & 0 & 0 & 0 & 0 & $X$ & 0 & 0 & 0 & 0 & 0 & 0 & 0 & 0 & 0 & $X$ & 0 & $x$ & 0 & 0 & 0 & 0 & 0 & 0 \\
\hline K16 & $\mathrm{X}$ & $X$ & $\mathrm{X}$ & $\mathrm{X}$ & $\bar{X}$ & $\bar{X}$ & $\bar{X}$ & $x$ & $\mathrm{X}$ & $x$ & $\bar{X}$ & $x$ & $x$ & 0 & 0 & $\bar{x}$ & $x$ & $x$ & 0 & 0 & $\bar{X}$ & $x$ & 0 & 0 & 0 & 0 & $\bar{X}$ & 0 & 0 & 0 & 0 & 0 & 0 & 0 & $\bar{X}$ & 0 & 0 & $x$ & 0 & 0 & $\bar{x}$ \\
\hline K17 & $x$ & $x$ & $x$ & $x$ & $X$ & $x$ & $\mathrm{X}$ & $X$ & $X$ & $x$ & $x$ & $X$ & $x$ & $\bar{X}$ & $\bar{X}$ & $\mathrm{X}$ & $X$ & $x$ & $\bar{X}$ & $\bar{X}$ & $X$ & $\mathrm{X}$ & $\bar{X}$ & 0 & 0 & $\bar{X}$ & $\bar{x}$ & 0 & $x$ & 0 & 0 & 0 & $x$ & 0 & 0 & 0 & 0 & $x$ & 0 & $x$ & $\mathrm{X}$ \\
\hline K18 & $x$ & $x$ & $x$ & $X$ & $x$ & $x$ & $x$ & $x$ & $x$ & $x$ & $x$ & $x$ & $x$ & $x$ & $x$ & $x$ & $x$ & 0 & 0 & $x$ & $x$ & $x$ & $x$ & $x$ & $\bar{x}$ & $\bar{x}$ & $\bar{x}$ & $x$ & 0 & $x$ & 0 & $x$ & $x$ & $x$ & 0 & 0 & $\bar{x}$ & 0 & $x$ & 0 & $x$ \\
\hline K19 & $X$ & $X$ & $X$ & $x$ & $X$ & $x$ & $X$ & $X$ & 0 & 0 & $x$ & $X$ & 0 & 0 & 0 & 0 & 0 & 0 & 0 & 0 & 0 & $X$ & 0 & 0 & 0 & 0 & 0 & 0 & 0 & 0 & 0 & $\bar{X}$ & 0 & 0 & 0 & 0 & 0 & 0 & 0 & 0 & $X$ \\
\hline K21 & $\mathrm{X}$ & $X$ & $\mathrm{X}$ & $\mathrm{X}$ & $X$ & $\mathrm{X}$ & $\bar{X}$ & $X$ & $X$ & $x$ & $x$ & 0 & 0 & 0 & 0 & 0 & 0 & 0 & 0 & 0 & 0 & 0 & 0 & 0 & 0 & 0 & 0 & 0 & 0 & 0 & 0 & 0 & 0 & 0 & 0 & 0 & 0 & 0 & 0 & 0 & $\bar{X}$ \\
\hline K22 & $x$ & $x$ & $x$ & $x$ & $X$ & $x$ & $\bar{x}$ & $X$ & $x$ & $x$ & $x$ & $x$ & $x$ & $X$ & $x$ & $\mathrm{X}$ & 0 & $x$ & $\bar{X}$ & $\bar{X}$ & $x$ & $\bar{X}$ & $\bar{X}$ & $\bar{X}$ & 0 & 0 & 0 & 0 & 0 & 0 & 0 & 0 & $x$ & 0 & 0 & 0 & 0 & $X$ & 0 & $x$ & $\bar{X}$ \\
\hline K23 & $X$ & $x$ & $x$ & $x$ & $X$ & $x$ & 0 & $x$ & 0 & $x$ & $X$ & 0 & 0 & 0 & $X$ & 0 & 0 & 0 & 0 & 0 & 0 & 0 & 0 & 0 & 0 & 0 & 0 & 0 & 0 & 0 & 0 & 0 & 0 & 0 & 0 & 0 & 0 & 0 & 0 & 0 & 0 \\
\hline K24 & $X$ & $X$ & $X$ & $\mathrm{X}$ & $X$ & $x$ & $\bar{X}$ & $X$ & $x$ & 0 & $x$ & $x$ & 0 & $x$ & 0 & 0 & 0 & 0 & $\bar{X}$ & 0 & $x$ & 0 & $\bar{x}$ & 0 & 0 & $\bar{X}$ & 0 & 0 & 0 & 0 & 0 & 0 & 0 & 0 & 0 & 0 & 0 & 0 & 0 & 0 & 0 \\
\hline K25 & $X$ & $X$ & $X$ & $X$ & $X$ & $\bar{X}$ & $\bar{X}$ & 0 & $X$ & 0 & 0 & 0 & 0 & 0 & 0 & 0 & 0 & $\bar{x}$ & 0 & 0 & 0 & 0 & 0 & 0 & 0 & 0 & 0 & 0 & 0 & 0 & 0 & 0 & 0 & 0 & 0 & 0 & 0 & 0 & 0 & 0 & $\bar{x}$ \\
\hline K26 & $\mathrm{X}$ & $X$ & $X$ & $\mathrm{X}$ & $X$ & $x$ & $X$ & $x$ & $X$ & $X$ & $x$ & $x$ & $x$ & $X$ & $x$ & $X$ & 0 & $X$ & $X$ & $x$ & $x$ & 0 & $x$ & $x$ & 0 & $\bar{x}$ & $\bar{x}$ & 0 & 0 & $X$ & $X$ & $\bar{X}$ & 0 & $X$ & 0 & 0 & $\bar{X}$ & 0 & 0 & 0 & $X$ \\
\hline K27 & $\mathrm{X}$ & $\mathrm{X}$ & $X$ & $x$ & $x$ & $x$ & $X$ & $X$ & 0 & 0 & 0 & 0 & $x$ & 0 & $x$ & 0 & 0 & $x$ & 0 & 0 & 0 & 0 & 0 & 0 & 0 & 0 & 0 & 0 & 0 & 0 & 0 & 0 & 0 & 0 & 0 & 0 & 0 & 0 & 0 & 0 & 0 \\
\hline K28 & $x$ & $x$ & $x$ & $x$ & 0 & $x$ & 0 & $x$ & 0 & 0 & 0 & 0 & 0 & 0 & $x$ & 0 & 0 & 0 & 0 & 0 & 0 & 0 & 0 & 0 & 0 & 0 & 0 & 0 & 0 & 0 & 0 & 0 & 0 & 0 & 0 & 0 & 0 & 0 & 0 & 0 & 0 \\
\hline K29 & $X$ & $X$ & $X$ & $x$ & $x$ & $x$ & 0 & 0 & 0 & $x$ & 0 & 0 & 0 & 0 & 0 & 0 & 0 & 0 & 0 & 0 & 0 & 0 & 0 & 0 & 0 & 0 & $\bar{X}$ & 0 & 0 & 0 & 0 & 0 & 0 & 0 & 0 & 0 & 0 & 0 & 0 & 0 & 0 \\
\hline K30 & $x$ & $x$ & $x$ & $X$ & $x$ & 0 & 0 & 0 & 0 & 0 & 0 & 0 & 0 & 0 & 0 & 0 & 0 & 0 & 0 & 0 & 0 & 0 & 0 & 0 & 0 & 0 & 0 & 0 & 0 & 0 & 0 & 0 & 0 & 0 & 0 & 0 & 0 & 0 & 0 & 0 & 0 \\
\hline K31 & $x$ & $X$ & $X$ & $x$ & $x$ & 0 & 0 & $x$ & 0 & 0 & 0 & $x$ & 0 & $x$ & 0 & 0 & $x$ & 0 & 0 & 0 & 0 & 0 & 0 & 0 & 0 & 0 & 0 & 0 & 0 & 0 & 0 & 0 & 0 & 0 & 0 & 0 & 0 & 0 & 0 & 0 & $\bar{X}$ \\
\hline K32 & $x$ & $x$ & $x$ & $x$ & $X$ & $x$ & $x$ & $X$ & 0 & 0 & $x$ & 0 & 0 & 0 & $x$ & 0 & 0 & $x$ & 0 & $x$ & 0 & 0 & 0 & 0 & 0 & 0 & $\bar{X}$ & 0 & 0 & $x$ & 0 & 0 & 0 & 0 & 0 & 0 & 0 & 0 & 0 & $x$ & $X$ \\
\hline K33 & $\mathrm{X}$ & $X$ & $\mathrm{X}$ & $X$ & $X$ & $\mathrm{X}$ & $X$ & $X$ & $X$ & 0 & 0 & 0 & 0 & 0 & 0 & 0 & 0 & 0 & 0 & $X$ & 0 & $x$ & 0 & 0 & 0 & 0 & 0 & 0 & 0 & 0 & 0 & 0 & 0 & 0 & 0 & 0 & 0 & 0 & 0 & 0 & 0 \\
\hline
\end{tabular}


Table 9. Cont

\begin{tabular}{|c|c|c|c|c|c|c|c|c|c|c|c|c|c|c|c|c|c|c|c|c|c|c|c|c|c|c|c|c|c|c|c|c|c|c|c|c|c|c|c|c|c|}
\hline Ranges & P1 & P2 & P3 & P4 & P5 & P6 & P7 & P8 & P9 & P10 & P11 & P12 & P13 & P14 & P15 & P16 & P17 & P18 & P19 & P20 & P21 & P22 & P23 & $\mathbf{P 2 4}$ & P25 & P26 & P27 & P28 & P29 & P30 & P31 & P32 & P33 & P34 & P35 & P36 & P37 & P38 & P39 & P40 & P41 \\
\hline \multicolumn{42}{|l|}{ Category } \\
\hline K34 & $\mathrm{x}$ & $x$ & $\mathrm{x}$ & $x$ & 0 & 0 & $\mathrm{x}$ & 0 & $x$ & 0 & 0 & 0 & 0 & 0 & 0 & 0 & 0 & 0 & 0 & 0 & 0 & 0 & 0 & 0 & 0 & 0 & 0 & 0 & 0 & 0 & 0 & 0 & 0 & 0 & 0 & 0 & 0 & 0 & 0 & 0 & 0 \\
\hline K35 & $X$ & $X$ & $X$ & $X$ & $x$ & $x$ & 0 & $x$ & $X$ & 0 & 0 & 0 & 0 & 0 & 0 & $X$ & 0 & $x$ & 0 & 0 & $x$ & 0 & $X$ & 0 & 0 & 0 & 0 & 0 & 0 & 0 & 0 & 0 & 0 & 0 & 0 & 0 & 0 & 0 & 0 & 0 & 0 \\
\hline K36 & $\mathrm{X}$ & $x$ & $x$ & $\mathrm{X}$ & $x$ & $x$ & 0 & $x$ & $\mathrm{x}$ & 0 & 0 & 0 & 0 & 0 & 0 & 0 & 0 & 0 & 0 & 0 & 0 & 0 & 0 & 0 & $\bar{x}$ & 0 & 0 & 0 & 0 & 0 & 0 & 0 & 0 & 0 & 0 & 0 & 0 & 0 & 0 & 0 & 0 \\
\hline K37 & $\mathrm{X}$ & $\mathrm{X}$ & $\mathrm{X}$ & $\mathrm{X}$ & 0 & $\mathrm{X}$ & $\mathrm{X}$ & 0 & 0 & 0 & 0 & 0 & 0 & 0 & 0 & 0 & 0 & 0 & 0 & 0 & 0 & 0 & 0 & 0 & 0 & 0 & 0 & 0 & 0 & 0 & 0 & 0 & 0 & 0 & 0 & 0 & 0 & 0 & 0 & 0 & 0 \\
\hline K38 & $X$ & $X$ & $X$ & 0 & $x$ & $x$ & $X$ & 0 & $X$ & 0 & 0 & 0 & 0 & 0 & $X$ & 0 & 0 & 0 & 0 & 0 & 0 & 0 & 0 & 0 & 0 & 0 & 0 & 0 & 0 & 0 & 0 & 0 & 0 & 0 & 0 & 0 & 0 & 0 & 0 & 0 & 0 \\
\hline K39 & $x$ & $x$ & $x$ & $x$ & $x$ & 0 & 0 & 0 & 0 & 0 & 0 & 0 & 0 & 0 & 0 & 0 & 0 & 0 & 0 & 0 & 0 & 0 & 0 & 0 & 0 & 0 & 0 & 0 & 0 & 0 & 0 & 0 & 0 & 0 & 0 & 0 & 0 & 0 & 0 & 0 & 0 \\
\hline K40 & $x$ & $x$ & $x$ & 0 & 0 & $x$ & 0 & 0 & 0 & 0 & 0 & 0 & 0 & 0 & 0 & 0 & 0 & 0 & 0 & 0 & 0 & 0 & 0 & 0 & 0 & 0 & 0 & 0 & 0 & 0 & 0 & 0 & 0 & 0 & 0 & 0 & 0 & 0 & 0 & 0 & 0 \\
\hline K41 & $X$ & $x$ & 0 & $X$ & 0 & $X$ & 0 & 0 & 0 & 0 & $\bar{X}$ & 0 & 0 & 0 & 0 & 0 & 0 & 0 & 0 & 0 & 0 & 0 & 0 & 0 & 0 & 0 & 0 & 0 & 0 & 0 & 0 & 0 & 0 & 0 & 0 & 0 & 0 & 0 & 0 & 0 & 0 \\
\hline
\end{tabular}

Table 10. KA matrix: project categories according to the duration of the campaign.

\begin{tabular}{|c|c|c|c|c|c|c|c|c|c|c|c|c|c|c|c|c|c|c|c|c|c|c|c|c|c|c|c|c|c|c|c|}
\hline Ranges & P1 & P2 & P3 & P4 & P5 & P6 & P7 & P8 & P9 & P10 & P11 & P12 & P13 & P14 & P15 & P16 & P17 & P18 & P19 & P20 & P21 & P22 & P23 & P24 & P25 & P26 & P27 & P28 & P29 & P30 & P31 \\
\hline \multicolumn{32}{|l|}{ Category } \\
\hline $\mathrm{K} 1$ & 0 & $x$ & $X$ & $x$ & $x$ & $x$ & $X$ & $X$ & $x$ & $X$ & $X$ & $X$ & $X$ & $X$ & $X$ & $X$ & $x$ & $X$ & $X$ & $X$ & $x$ & $x$ & $x$ & $x$ & $X$ & $x$ & $X$ & $x$ & $X$ & $x$ & $x$ \\
\hline $\mathrm{K} 2$ & $x$ & $x$ & $x$ & $x$ & $x$ & $x$ & $x$ & $X$ & $x$ & $x$ & $x$ & $x$ & $x$ & $x$ & $X$ & $X$ & $X$ & $X$ & $X$ & $X$ & $X$ & $X$ & $X$ & $x$ & $x$ & $x$ & $X$ & $x$ & $x$ & $x$ & $x$ \\
\hline K3 & $x$ & $x$ & $x$ & $x$ & $x$ & $x$ & $x$ & $X$ & $x$ & $X$ & $X$ & $x$ & $x$ & $x$ & $x$ & $x$ & $X$ & $x$ & $x$ & $x$ & $X$ & $x$ & $x$ & $x$ & $X$ & $x$ & $X$ & $x$ & $x$ & $x$ & $x$ \\
\hline K4 & $x$ & $x$ & $x$ & $x$ & $x$ & $x$ & $x$ & $X$ & $x$ & $X$ & $X$ & $x$ & $X$ & $x$ & $X$ & $x$ & $x$ & $x$ & $x$ & $x$ & $X$ & $x$ & $x$ & $X$ & $x$ & $x$ & $x$ & $x$ & $x$ & $x$ & 0 \\
\hline K5 & $x$ & $x$ & $x$ & $x$ & $X$ & $x$ & $x$ & $X$ & $x$ & $X$ & $X$ & $x$ & $x$ & $x$ & $X$ & $x$ & $X$ & $X$ & $X$ & $X$ & $X$ & $X$ & $x$ & $X$ & $X$ & $x$ & $X$ & $x$ & $X$ & $X$ & $x$ \\
\hline K6 & $x$ & $x$ & $x$ & $x$ & $x$ & $x$ & $x$ & $X$ & $x$ & $X$ & $X$ & $X$ & $X$ & $x$ & $x$ & $X$ & $X$ & $X$ & $X$ & $X$ & $X$ & $X$ & $X$ & 0 & $X$ & 0 & 0 & 0 & 0 & $X$ & 0 \\
\hline K7 & $X$ & $x$ & $X$ & $x$ & $X$ & $x$ & $X$ & $X$ & $x$ & $X$ & $X$ & $X$ & $X$ & $X$ & $X$ & $X$ & $X$ & $X$ & $X$ & $X$ & $X$ & $X$ & $X$ & $X$ & $X$ & $x$ & $X$ & $x$ & $X$ & $X$ & $X$ \\
\hline K8 & 0 & 0 & $X$ & $x$ & $X$ & $x$ & $X$ & $X$ & $X$ & $X$ & $X$ & $X$ & $X$ & $X$ & $X$ & $X$ & $X$ & $X$ & $X$ & $X$ & $X$ & $X$ & $X$ & $X$ & $X$ & $x$ & $X$ & 0 & 0 & $x$ & $x$ \\
\hline K9 & $X$ & $X$ & $X$ & $X$ & $X$ & $X$ & $X$ & $X$ & $X$ & $X$ & $X$ & $X$ & $X$ & $X$ & $X$ & $X$ & $X$ & $X$ & $X$ & $X$ & $X$ & $X$ & $X$ & $X$ & $x$ & $X$ & $X$ & $X$ & 0 & $X$ & $X$ \\
\hline K10 & $x$ & 0 & $x$ & $x$ & $X$ & $x$ & $X$ & $x$ & $x$ & $x$ & $x$ & $X$ & $X$ & $X$ & $X$ & $x$ & $x$ & $X$ & $x$ & $X$ & $X$ & $X$ & $X$ & 0 & $x$ & $X$ & $x$ & 0 & $X$ & $x$ & 0 \\
\hline K11 & 0 & $x$ & $X$ & $x$ & $X$ & $x$ & $X$ & $X$ & $x$ & $X$ & $x$ & $X$ & $X$ & $X$ & $X$ & $X$ & $X$ & $X$ & $X$ & $X$ & $X$ & $X$ & $X$ & $X$ & $x$ & $X$ & 0 & $x$ & 0 & $X$ & 0 \\
\hline K12 & $X$ & $X$ & $X$ & $X$ & $X$ & $X$ & $X$ & $X$ & $x$ & $X$ & $X$ & $X$ & $X$ & $X$ & $X$ & $X$ & $X$ & $X$ & $X$ & $X$ & $X$ & $X$ & $X$ & $X$ & $X$ & 0 & 0 & 0 & 0 & $X$ & 0 \\
\hline
\end{tabular}


Table 10. Cont.

\begin{tabular}{|c|c|c|c|c|c|c|c|c|c|c|c|c|c|c|c|c|c|c|c|c|c|c|c|c|c|c|c|c|c|c|c|}
\hline Ranges & P1 & P2 & P3 & P4 & P5 & P6 & P7 & P8 & P9 & P10 & P11 & P12 & P13 & P14 & P15 & P16 & P17 & P18 & P19 & P20 & P21 & $\mathrm{P} 22$ & $\mathbf{P} 23$ & $\mathrm{P} 24$ & P25 & P26 & P27 & P28 & P29 & P30 & P31 \\
\hline \multicolumn{32}{|l|}{ Category } \\
\hline K13 & $X$ & $X$ & $X$ & $X$ & $X$ & $X$ & $X$ & $X$ & $X$ & $X$ & $X$ & $X$ & $x$ & $X$ & $X$ & $X$ & $X$ & $x$ & $X$ & $x$ & $x$ & $x$ & $X$ & $x$ & $X$ & $X$ & $X$ & $X$ & $X$ & $X$ & $X$ \\
\hline K14 & $x$ & $x$ & $x$ & $x$ & $x$ & $x$ & $x$ & $x$ & $x$ & $x$ & $x$ & $x$ & $x$ & $x$ & $x$ & $x$ & $x$ & $x$ & $x$ & $x$ & $x$ & $x$ & $x$ & $x$ & $x$ & $x$ & 0 & $X$ & $x$ & $x$ & $X$ \\
\hline K15 & $X$ & 0 & $X$ & 0 & $X$ & $X$ & $X$ & $X$ & $X$ & $X$ & $x$ & $X$ & $x$ & $X$ & $X$ & $X$ & $x$ & $X$ & $x$ & $x$ & $X$ & $x$ & $x$ & $x$ & $x$ & $X$ & 0 & $X$ & $x$ & $X$ & 0 \\
\hline K16 & $X$ & $X$ & $X$ & $X$ & $X$ & $X$ & $X$ & $X$ & $X$ & $X$ & $X$ & $X$ & $X$ & $X$ & $X$ & $X$ & $X$ & $X$ & $X$ & $x$ & $x$ & $X$ & $X$ & $X$ & $X$ & 0 & $X$ & $X$ & $X$ & $X$ & 0 \\
\hline K17 & $X$ & 0 & 0 & 0 & $X$ & $X$ & $X$ & $X$ & 0 & $X$ & $X$ & $X$ & $X$ & $X$ & $X$ & $X$ & $X$ & $X$ & $x$ & $x$ & $X$ & 0 & $X$ & $X$ & $X$ & 0 & 0 & 0 & 0 & $X$ & 0 \\
\hline K19 & $X$ & $X$ & 0 & $X$ & $X$ & $X$ & $X$ & $X$ & $X$ & $X$ & $X$ & $X$ & $X$ & $X$ & $X$ & $X$ & $X$ & $X$ & $x$ & $x$ & $x$ & $X$ & $X$ & $X$ & $X$ & $X$ & $X$ & 0 & $X$ & $x$ & 0 \\
\hline K20 & $X$ & 0 & $X$ & $X$ & $X$ & 0 & $X$ & $x$ & $x$ & $X$ & $X$ & $X$ & $X$ & $X$ & $X$ & $X$ & $x$ & $X$ & $x$ & $x$ & $x$ & $X$ & $X$ & 0 & $X$ & 0 & 0 & 0 & 0 & $X$ & $X$ \\
\hline K21 & $X$ & 0 & $X$ & $X$ & $X$ & $X$ & $X$ & $X$ & $X$ & $X$ & $X$ & $X$ & $X$ & $X$ & $X$ & $X$ & $X$ & $X$ & $X$ & $X$ & $X$ & 0 & $X$ & 0 & $X$ & 0 & 0 & 0 & 0 & $X$ & 0 \\
\hline K22 & 0 & 0 & 0 & $X$ & $X$ & $X$ & $X$ & $X$ & $X$ & $X$ & $X$ & $X$ & $X$ & $X$ & $X$ & $X$ & $X$ & $X$ & $x$ & $X$ & 0 & 0 & $X$ & 0 & $X$ & $X$ & 0 & 0 & 0 & $X$ & 0 \\
\hline K23 & $X$ & 0 & 0 & $X$ & $X$ & $X$ & $X$ & $X$ & $X$ & $X$ & $X$ & $X$ & $X$ & $X$ & $X$ & $X$ & $X$ & $X$ & 0 & $X$ & $x$ & $X$ & 0 & 0 & 0 & 0 & 0 & 0 & 0 & $X$ & 0 \\
\hline K24 & $X$ & $X$ & 0 & $X$ & $X$ & $X$ & $X$ & $x$ & $x$ & $X$ & $X$ & $X$ & $X$ & $x$ & $X$ & $X$ & $x$ & $X$ & 0 & $x$ & $x$ & 0 & $X$ & $X$ & $X$ & 0 & 0 & $X$ & $X$ & $x$ & 0 \\
\hline K25 & $X$ & 0 & 0 & 0 & 0 & 0 & $X$ & $X$ & 0 & 0 & $X$ & $x$ & $X$ & $X$ & $X$ & $X$ & 0 & $X$ & $x$ & 0 & $X$ & 0 & $X$ & 0 & 0 & 0 & 0 & 0 & 0 & $x$ & 0 \\
\hline K27 & 0 & 0 & 0 & 0 & $X$ & $X$ & $X$ & $X$ & $X$ & $X$ & $X$ & $X$ & $X$ & $X$ & $X$ & $X$ & $X$ & $X$ & $X$ & $X$ & $x$ & $X$ & $X$ & 0 & 0 & 0 & 0 & 0 & 0 & $X$ & 0 \\
\hline K28 & $X$ & 0 & 0 & $X$ & $X$ & 0 & $X$ & $x$ & $X$ & $X$ & $X$ & 0 & $X$ & $X$ & $X$ & $X$ & 0 & $X$ & 0 & $X$ & $x$ & 0 & $X$ & 0 & $X$ & $X$ & 0 & 0 & 0 & $x$ & 0 \\
\hline K29 & $X$ & $X$ & 0 & 0 & 0 & 0 & $X$ & $X$ & $x$ & $X$ & $X$ & 0 & $X$ & $X$ & $X$ & $X$ & $X$ & 0 & 0 & $X$ & 0 & 0 & $X$ & 0 & $X$ & 0 & 0 & 0 & $X$ & $X$ & 0 \\
\hline K30 & 0 & 0 & 0 & 0 & $X$ & 0 & 0 & $x$ & $x$ & $x$ & 0 & $x$ & $X$ & $X$ & $X$ & $X$ & $x$ & $X$ & $X$ & $X$ & 0 & 0 & $X$ & 0 & 0 & 0 & 0 & 0 & 0 & $X$ & 0 \\
\hline K31 & $X$ & 0 & $X$ & 0 & $x$ & $X$ & $X$ & $x$ & $x$ & $x$ & $x$ & $x$ & 0 & $x$ & $x$ & $x$ & $x$ & 0 & 0 & $x$ & 0 & 0 & 0 & 0 & 0 & 0 & 0 & 0 & 0 & $x$ & 0 \\
\hline K32 & $X$ & 0 & 0 & 0 & 0 & 0 & $x$ & 0 & $X$ & 0 & $X$ & $X$ & 0 & 0 & $X$ & $X$ & $X$ & $X$ & $x$ & $x$ & 0 & 0 & $X$ & $X$ & $X$ & 0 & 0 & 0 & 0 & $x$ & $X$ \\
\hline K33 & 0 & 0 & 0 & 0 & 0 & 0 & $X$ & 0 & 0 & $X$ & $X$ & $X$ & $X$ & $X$ & $X$ & 0 & 0 & $X$ & 0 & $x$ & 0 & 0 & 0 & 0 & $X$ & 0 & 0 & 0 & 0 & $x$ & 0 \\
\hline K34 & $X$ & 0 & 0 & $X$ & 0 & 0 & $X$ & $x$ & 0 & $X$ & $X$ & $x$ & $X$ & $X$ & $X$ & 0 & 0 & $X$ & 0 & 0 & 0 & 0 & 0 & 0 & 0 & 0 & 0 & 0 & 0 & 0 & 0 \\
\hline K35 & 0 & 0 & 0 & 0 & 0 & 0 & 0 & $x$ & 0 & $x$ & $x$ & $x$ & 0 & 0 & $x$ & $X$ & $X$ & $x$ & 0 & 0 & 0 & 0 & 0 & 0 & 0 & $X$ & 0 & 0 & 0 & $x$ & 0 \\
\hline
\end{tabular}


Table 10. Cont.

\begin{tabular}{|c|c|c|c|c|c|c|c|c|c|c|c|c|c|c|c|c|c|c|c|c|c|c|c|c|c|c|c|c|c|c|c|}
\hline Ranges & P1 & P2 & P3 & P4 & P5 & P6 & P7 & P8 & P9 & P10 & P11 & P12 & P13 & P14 & P15 & P16 & P17 & P18 & P19 & P20 & P21 & P22 & P23 & P24 & P25 & P26 & P27 & P28 & P29 & P30 & P31 \\
\hline \multicolumn{32}{|l|}{ Category } \\
\hline K36 & 0 & 0 & 0 & 0 & 0 & 0 & $X$ & 0 & 0 & 0 & $X$ & 0 & 0 & $X$ & $X$ & $X$ & $X$ & 0 & $X$ & $X$ & $X$ & 0 & 0 & 0 & 0 & 0 & 0 & 0 & 0 & $X$ & 0 \\
\hline K37 & 0 & 0 & 0 & $X$ & 0 & 0 & 0 & 0 & $X$ & 0 & $X$ & 0 & 0 & $X$ & $X$ & 0 & 0 & 0 & $x$ & 0 & $x$ & 0 & 0 & 0 & 0 & 0 & 0 & 0 & 0 & $x$ & 0 \\
\hline K38 & 0 & 0 & 0 & 0 & 0 & 0 & $X$ & 0 & 0 & 0 & 0 & 0 & 0 & 0 & $x$ & $X$ & 0 & $X$ & $X$ & 0 & 0 & 0 & 0 & 0 & 0 & 0 & 0 & 0 & 0 & $x$ & 0 \\
\hline K39 & 0 & 0 & 0 & 0 & 0 & 0 & 0 & 0 & 0 & 0 & 0 & 0 & $x$ & $X$ & $X$ & 0 & 0 & 0 & 0 & 0 & 0 & 0 & $X$ & $X$ & 0 & 0 & 0 & 0 & 0 & $X$ & 0 \\
\hline K40 & 0 & 0 & 0 & 0 & 0 & 0 & 0 & 0 & 0 & 0 & 0 & 0 & 0 & 0 & 0 & 0 & 0 & 0 & 0 & 0 & 0 & 0 & 0 & 0 & 0 & 0 & 0 & 0 & 0 & 0 & $X$ \\
\hline K41 & 0 & 0 & 0 & 0 & 0 & 0 & 0 & $X$ & 0 & 0 & 0 & 0 & 0 & 0 & $X$ & 0 & 0 & 0 & 0 & 0 & 0 & 0 & $X$ & 0 & $X$ & 0 & 0 & 0 & 0 & 0 & 0 \\
\hline K42 & $x$ & 0 & $X$ & 0 & 0 & 0 & 0 & 0 & 0 & 0 & 0 & 0 & 0 & 0 & $X$ & 0 & 0 & $X$ & 0 & 0 & 0 & 0 & 0 & 0 & 0 & 0 & 0 & 0 & 0 & 0 & 0 \\
\hline
\end{tabular}

Table 11. KA matrix, in terms of project categories according to profitability.

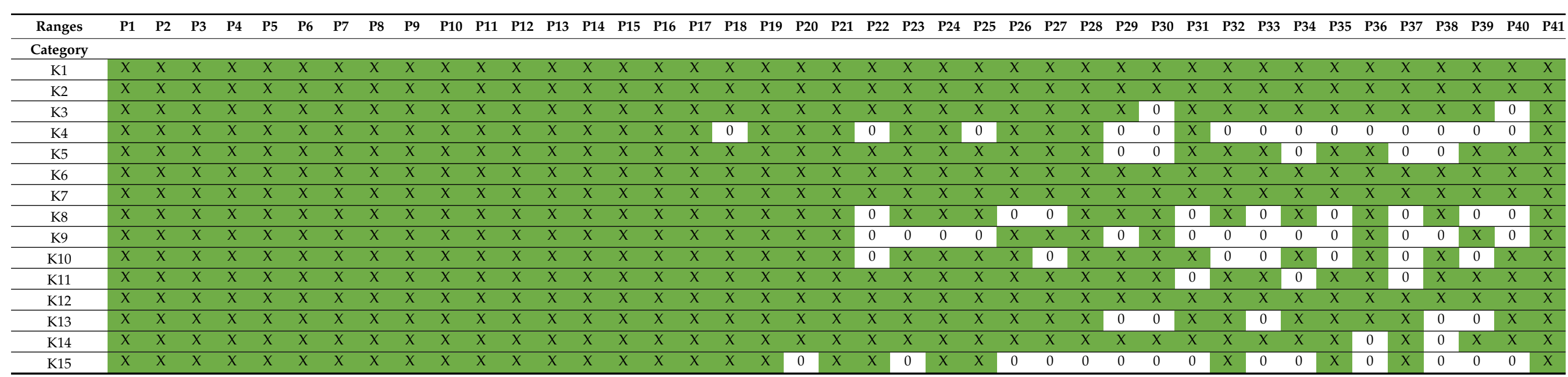


Table 11. Cont.

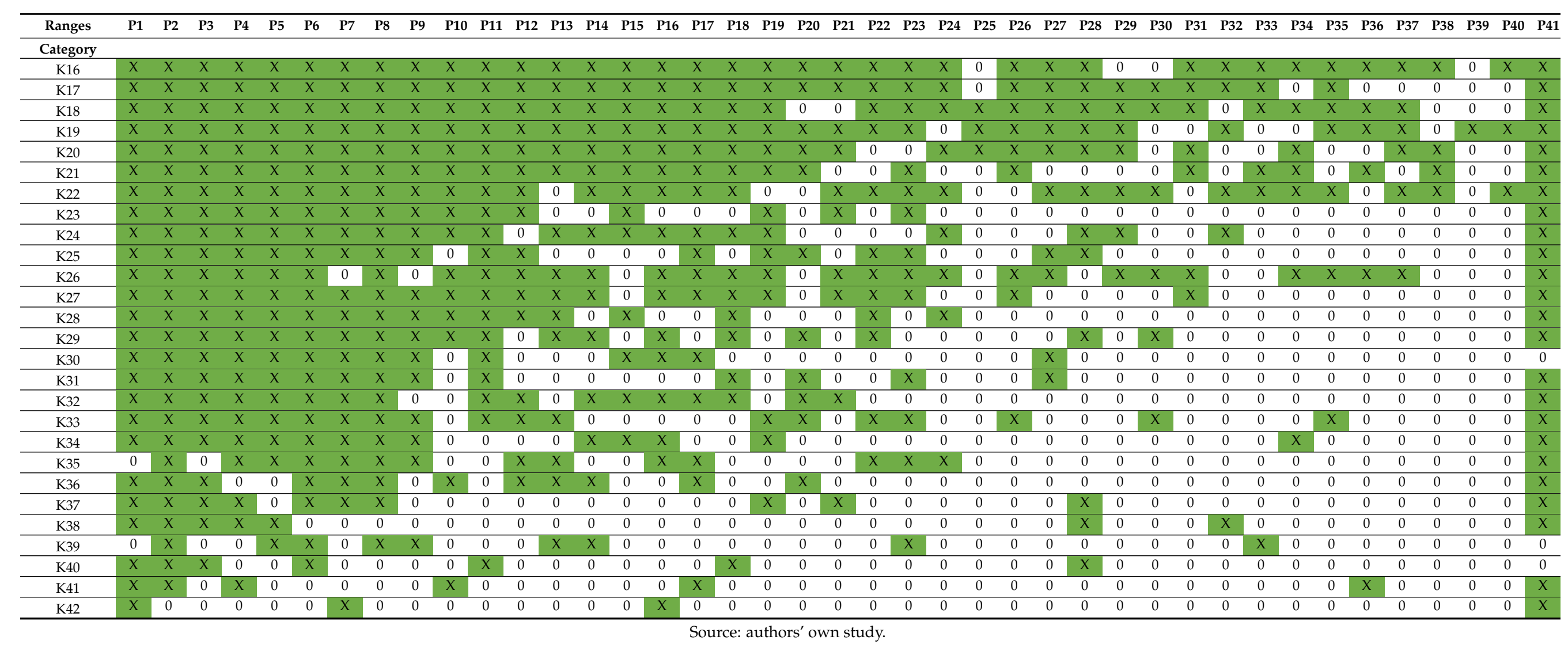


The specified 42 detailed categories of projects (Table 9) were grouped into six classes of similar categories after carrying out the classification procedure:

- $\mathrm{KLP} 1=\{\mathrm{K} 14, \mathrm{~K} 25, \mathrm{~K} 27, \mathrm{~K} 28, \mathrm{~K} 29, \mathrm{~K} 30, \mathrm{~K} 42\}$,

- $\mathrm{KLP} 2=\{\mathrm{K} 6, \mathrm{~K} 9, \mathrm{~K} 10, \mathrm{~K} 17, \mathrm{~K} 23, \mathrm{~K} 26\}$,

- $\mathrm{KLP} 3=\{\mathrm{K} 2, \mathrm{~K} 5, \mathrm{~K} 12, \mathrm{~K} 20, \mathrm{~K} 24, \mathrm{~K} 36\}$,

- $\mathrm{KLP} 4=\{\mathrm{K} 1, \mathrm{~K} 15, \mathrm{~K} 16, \mathrm{~K} 22, \mathrm{~K} 31, \mathrm{~K} 40, \mathrm{~K} 41\}$,

- $\mathrm{KLP5}=\{\mathrm{K} 4, \mathrm{~K} 7, \mathrm{~K} 13, \mathrm{~K} 18, \mathrm{~K} 19, \mathrm{~K} 32, \mathrm{~K} 34, \mathrm{~K} 35, \mathrm{~K} 39\}$,

- $\mathrm{KLP6}=\{\mathrm{K} 3, \mathrm{~K} 8, \mathrm{~K} 11, \mathrm{~K} 21, \mathrm{~K} 33, \mathrm{~K} 37, \mathrm{~K} 38\}$.

When analyzing the six classes of project categories according to the number of backers, it can be noticed that the number of categories in a given class is similar, and it ranges from seven to nine categories; that is, the number of supporters in the established ranges is similar. On the other hand, categories grouped by this attribute formed classes that were quite loosely related to each other thematically, for example KLP4 $=\{\mathrm{K} 1$ (comics), K15 (conceptual art), K16 (photography), K22 (playing cards), K31 (painting), K40 (video art), K41 (mobile games) $\}$ or KLP6 = \{K3 (classical music), K8 (art), K11 (illustrations), K21 (nature), K33 (translations), K37 (social practice) and K38 (performance art)\}.

The specified 42 detailed categories of projects (Table 10) were grouped into five classes of similar categories after carrying out the classification procedure:

- $\mathrm{KLT1}=\{\mathrm{K} 17, \mathrm{~K} 19, \mathrm{~K} 20, \mathrm{~K} 23, \mathrm{~K} 24, \mathrm{~K} 27, \mathrm{~K} 30, \mathrm{~K} 34\}$,

- $\mathrm{KLT} 2=\{\mathrm{K} 15, \mathrm{~K} 16, \mathrm{~K} 18, \mathrm{~K} 21, \mathrm{~K} 22\}$,

- $\mathrm{KLT3}=\{\mathrm{K} 28, \mathrm{~K} 31, \mathrm{~K} 32, \mathrm{~K} 33, \mathrm{~K} 35, \mathrm{~K} 36, \mathrm{~K} 37, \mathrm{~K} 38, \mathrm{~K} 39, \mathrm{~K} 40, \mathrm{~K} 41\}$,

- $\mathrm{KLT} 4=\{\mathrm{K} 1, \mathrm{~K} 2, \mathrm{~K} 3, \mathrm{~K} 4, \mathrm{~K} 5, \mathrm{~K} 7, \mathrm{~K} 8, \mathrm{~K} 9, \mathrm{~K} 10, \mathrm{~K} 11, \mathrm{~K} 12, \mathrm{~K} 13, \mathrm{~K} 14, \mathrm{~K} 25, \mathrm{~K} 42\}$,

- $\mathrm{KLT5}=\{\mathrm{K} 6, \mathrm{~K} 26, \mathrm{~K} 29\}$.

The project categories, classified according to the campaign duration, created five classes with greater than previously numerical variation within the class, namely from 3 to 15 categories. The thematic connection within classes is greatest in KLT5 $=\{$ K6 (board games), K26 (video games), K29 (romances) $\}$, and the weakest in KLT1 $=\{\mathrm{K} 17$ (science fiction), K19 (ceramics), K20 (people), K23 (digital art), K24 (animals), K27 (spaces), K30 (workshops) and K34 (sculpture)\}.

The specified 42 detailed categories of projects (Table 11) were grouped into five classes of similar categories after carrying out the classification procedure:

- $\mathrm{KLF} 1=\{\mathrm{K} 6, \mathrm{~K} 12, \mathrm{~K} 18, \mathrm{~K} 22, \mathrm{~K} 26, \mathrm{~K} 27, \mathrm{~K} 32, \mathrm{~K} 35, \mathrm{~K} 38, \mathrm{~K} 41, \mathrm{~K} 42\}$,

- $\mathrm{KLF} 2=\{\mathrm{K} 1, \mathrm{~K} 2, \mathrm{~K} 7, \mathrm{~K} 17, \mathrm{~K} 24, \mathrm{~K} 34\}$,

- $\mathrm{KLF} 3=\{\mathrm{K} 3, \mathrm{~K} 4, \mathrm{~K} 5, \mathrm{~K} 19, \mathrm{~K} 30, \mathrm{~K} 33\}$,

- $\mathrm{KLF} 4=\{\mathrm{K} 11, \mathrm{~K} 14, \mathrm{~K} 16, \mathrm{~K} 21, \mathrm{~K} 31, \mathrm{~K} 37\}$,

- $\quad \mathrm{KLF} 5=\{\mathrm{K} 8, \mathrm{~K} 9, \mathrm{~K} 10, \mathrm{~K} 13, \mathrm{~K} 15, \mathrm{~K} 20, \mathrm{~K} 23, \mathrm{~K} 25, \mathrm{~K} 28, \mathrm{~K} 29, \mathrm{~K} 36, \mathrm{~K} 39, \mathrm{~K} 40\}$.

When analyzing five classes of project categories according to profitability, it can be noticed that the number of categories in a given class is different than in the case of the previous attributes, as it ranges from 6 to 13 categories, with three out of seven classes having an equal number of categories (six each). The thematic scope is quite varied in all classes, for example KLF3 $=\{\mathrm{K} 3$ (classical music), K4 (performances), K5 (dance), K19 (ceramics), K30 (workshops) and K33 (translations)\}.

The results of the cross-interaction of the grouped classes are shown in Tables 12-14. The following ranges were included:

- $\quad>0.9$-very strong correlation,

- $0.7-0.9-$ quite strong correlation,

- $\quad 0.4-0.7-$ moderate correlation,

- 0.2-0.4-weak correlation,

- $<0.2-$ no correlation. 
Table 12. Results of the similarity study on a cross-basis of the class set KLF-KLT.

\begin{tabular}{cccccc}
\hline & KLT1 & KLT2 & KLT3 & KLT4 & KLT5 \\
\hline KLF1 & 1 & 2 & 4 & 2 & 2 \\
\hline KLF2 & 3 & 0 & 0 & 3 & 0 \\
\hline KLF3 & 2 & 0 & 2 & 2 & 0 \\
\hline KLF4 & 0 & 2 & 4 & & 1 \\
\hline KLF5 & 2 & 1 & & & \\
\hline & 0.28 & & & & \\
\hline & -0.88 & 0.91 & 0.19 & -0.22 & -0.41 \\
\hline & -0.94 & 0.77 & -0.46 & -0.92 & -0.51 \\
\hline
\end{tabular}

Source: authors' own study.

Table 13. The results of the similarity study on a cross-basis of the class set KLP-KLT.

\begin{tabular}{cccccc}
\hline & KLT1 & KLT2 & KLT3 & KLT4 & KLT5 \\
\hline KLP1 & 2 & 0 & 1 & 2 & 1 \\
\hline KLP2 & 2 & 0 & 0 & 2 & 2 \\
\hline KLP3 & 2 & 0 & 1 & 3 & 0 \\
\hline KLP4 & 0 & 3 & 3 & 3 & 0 \\
\hline KLP5 & 2 & 1 & 3 & & 0 \\
\hline KLP6 & 0 & 1 & & & \\
\hline & -0.53 & 0.56 & 0.34 & 0.12 & -0.3 \\
\hline & -0.41 & 0.62 & -0.64 & -0.41 & -0.41 \\
\hline & 0.15 & -0.33 & -0.06 & -0.28 & \\
\hline & -0.51 & 0.26 & 0.43 & &
\end{tabular}

Source: authors' own study.

The following sets of similar classes were obtained according to the strength of the correlation:

- $\quad>0.9=\{$ KLF2-KLT2 $\}$,

- $0.7-0.9=\{$ KLF3-KLT2 $\}$,

- $\quad 0.4-0.7=\{\}$,

- $0.2-0.4=\{$ KLF1-KLT1 $\}$,

- $\quad<0.2=\{K L F 2-K L T 1$, KLF3-KLT1, KLF4-KLT1, KLF5-KLT1, KLF4-KLT2, KLF5-KLT2, KLF3-KLT3, KLF4-KLT3, KLF5-KLT3, KLF4-KLT4, KLF5-KLT4, KLF5-KLT5\}.

After carrying out subsequent classification procedures, it was found that the profitability and campaign duration attributes have similar category classes with the correlation strength $>0.9$ and $0.7-0.9$ in two cases. However, most of these tandems, namely eighteen, have the correlation strength of $<0.2$. 
Table 14. Results of the similarity study on a cross-basis of the class set KLP-KLF.

\begin{tabular}{cccccc}
\hline & KLF1 & KLF2 & KLF3 & KLF4 & KLF5 \\
\hline KLP1 & 2 & 0 & 1 & 1 & 3 \\
\hline KLP2 & 2 & 2 & 0 & 0 & 3 \\
\hline KLP3 & 1 & 2 & 1 & 0 & 2 \\
\hline KLP4 & 2 & 0 & 0 & 3 & 2 \\
\hline KLP5 & 3 & 2 & 2 & & \\
\hline KLP6 & 1 & 0 & 2 & & \\
\hline & 0.62 & 0.27 & & 0.61 & 0.58 \\
\hline & 0.79 & 0.76 & 0.43 & -0.61 & -0.72 \\
\hline & 0.42 & -0.67 & 0.33 & 0.69 & \\
\hline
\end{tabular}

Source: authors' own study.

The following sets of similar classes were obtained according to the strength of the correlation:

- $>0.9=\{\}$,

- $0.7-0.9=\{\}$,

- $\quad 0.4-0.7=\{$ KLP2-KLT2, KLP3-KLT2, KLP6-KLT2 $\}$,

- $0.2-0.4=\{$ KLP5-KLT2, KLP3-KLT3 $\}$,

- $\quad<0.2=\{$ KLP1-KLT1, KLP2-KLT1, KLP3-KLT1, KLP4-KLT1, KLP5-KLT1, KLP4-KLT2, KLP4KLT3, KLP5-KLT3, KLP6-KLT3, KLP4-KLT4, KLP5-KLT4, KLP6-KLT4, KLP6-KLT5\}.

The following attributes were used: the number of backers and the campaign duration were grouped - as a result of carrying out the classification procedure-into similar classes of categories with the correlation strength of 0.4-0.7 and 0.2-0.4 (five tandems) and $<0.2$ (thirteen tandems). The study has shown that there was no great correlation between these attributes, as the ranges $>0.9$ and $0.7-0.9$ are empty sets.

The following sets of similar classes were obtained according to the strength of the relationships:

- $>0.9=\{\}$,

- $0.7-0.9=\{$ KLP2-KLF1, KLP3-KLF2 $\}$,

- $\quad 0.4-0.7=\{K L P 1-K L F 1, K L P 3-K L F 1, K L P 4-K L F 1, K L P 3-K L F 3, K L P 5-K L F 3, K L P 4-K L F 4$, KLP6-KLF4, KLP5-KLF5\},

- $\quad 0.2-0.4=\{\mathrm{KLP} 2-\mathrm{KLF} 2, \mathrm{KLP} 5-\mathrm{KLF} 2, \mathrm{KLP} 4-\mathrm{KLF} 3\}$,

- $\quad<0.2=\{K L P 5-K L F 1, K L P 6-K L F 1$, KLP4-KLF2, KLP6-KLF2, KLP6-KLF3, KLP5-KLF4, KLP6-KLF5\}.

On the other hand, the correlation between the attributes of the number of backers and project profitability turned out to be quite strong and moderately strong in ten cases, and weak in three compared classes. No correlation occurred in seven cases.

\section{Discussion}

The literature review has allowed for a conclusion that certain attributes of crowdfunding were separately analyzed in a few studies. It is true that some elements of taxonomy and clustering methods used to analyze and forecast the success or failure of projects are contained in the abovementioned paper by Fernandez-Blanco et al. [63], but no clear-cut solution has been presented for examining the relationships of attributes in terms of similarity or classification of their groups, which were created by taking into account various criteria. 
The results of this study can be viewed as a supplement to the research presented by Usman et al. [61] concerning the problem of information asymmetry in decision-making processes in crowdfunding. As already mentioned, the lack of "fairly" available information is particularly noticeable here, and when such a situation occurs, decisions can be highly influenced by emotions or the first impressions, as discussed by Schraven et al. [64]. The analysis and classification of crowdfunding attributes presented in this paper provides a large amount of information that may constitute the basis for more rational decisions, especially taking into account that the research results are presented in various crosssections, and the obtained category classes of projects or attributes make it possible to capture their similarity and the strength of correlation.

Therefore, it can be concluded that the purpose of this study was achieved, and the proposed approach with the use of the classification method, as well as the results of crowdfunding research, contributes to the current state of knowledge in the field of behavioral economics. These results may be helpful in the decision-making process, which is influenced by emotions, certain sympathies or preferences, especially in a situation of information asymmetry.

The aim of the article was to analyze selected crowdfunding attributes: project categories, the number of people backing the project, the duration of the campaign, the profitability of the project and the rewards offered, as well as their mutual classification, i.e., the creation of classes of similar attributes.

The first part of the research concerned establishing the names of the main and detailed categories by extracting them from the general fields of the source data. The remaining attributes were analyzed in terms of data availability and comparability in the projects. At this stage, one attribute was eliminated from further stages of the research, namely backer rewards, since this attribute did not meet the condition of comparability. Then, the value ranges of the attributes were specified by analyzing the various distributions of their values multiple times.

The numerical data prepared in this way were subjected to the classification procedure, developed in accordance with the specifics of dataset research, and three groups of project category classes were obtained, and they are similar in terms of each of the three attributes.

The second part of the research concerned the cross-correlations within the created category classes, but according to different attributes and the determination of the strength of their dependence on each other.

As it has already been emphasized, the results of this study, apart from the scientific aspect, also have a practical application. This is important because the information provided by platform owners is, in many cases, insufficient for the participants in the crowdfunding process, i.e., for the platform owners themselves, the creators of projects posted on them and for people backing projects or considering such a possibility, including potential investors.

For example, the Kickstarter platform makes information available by presenting it according to the following criteria:

- Popularity of the project,

- The number of people backing the project,

- Duration of the campaign of a given project (ending or newest),

- Geographically closest projects that can be supported,

- The highest-financed projects,

- The most frequently financed projects (according to the number of donors),

- Project status (active, successful, failed and canceled).

There are, however, no analyses or exploration of knowledge helpful for the participants of the crowdfunding process. It is easy to notice that a vast majority of these are very detailed pieces of information, provided at the level of individual projects. On an average day there are tens of thousands projects with the "active" status only on this one studied platform. As it transpires from the analyzed literature [36-39], the platform owners themselves, despite the use of robots crawling this immense amount of data, would be 
interested in information provided in various cross-sections and at different levels of detail, which would encourage both project creators and backers (including investors) to choose this platform, which in turn could entail financial success. The research results presented here, and especially the methodology of obtaining them, for example every day or week, could significantly improve the information offer of the platform (Table 3).

The aforementioned investors, who, unlike a large group of backers, want to generate income on their-hopefully correct-decision to invest in a group of projects, will find in the presented overviews information about the categories of projects that quickly become financially successful, about the correlation (defined on a five-point scale) between the amount of money collected and the duration of the campaign (Table 12) or the strength of the correlation between the duration and the number of people backing projects in a given category (Table 13). These data can also be obtained from a historical perspective by repeating selected classifications at desired intervals, which is of particular importance in strategic decisions.

The project creator, on the basis of selected overviews, can choose the category to which they want to submit their project, not only on the basis of its name but also on the information whether it is in a class supported by a significant number of people (Table 13) or in the one where the projects become financially successful the fastest (Tables 7 and 14). Of course, the name of the category should reflect the contents of the specific project, but the owner can choose, for example, between Category 8 (art) or 31 (painting) or between Category 20 (people) or 37 (social activity).

In-depth knowledge exploration may also be very helpful for project creators in determining the desired duration of the campaign or financing tiers (which should obviously be performed in accordance with the planned project budget), and also in deciding what type of financing to allow, e.g., flexible or fixed (Table 7). Project backers will be able to assess their decisions, for instance, taking into account the duration of the campaign (Table 6), which categories of projects achieve financial success in similar time periods (Tables 7 and 10), or which were supported by similarly sized groups of people (Tables 5 and 11). Moreover, when going to the project level within a category (which is possible thanks to the presented methodology), it would be possible to compare thematically similar projects according to selected attributes.

\section{Conclusions}

It should be noted that the analysis of crowdfunding attributes and the course of the classification procedure have allowed to confirm the first research hypothesis with regard to four selected attributes - project category, the number of backers, campaign duration and project profitability - since after determining their weights, it was possible to group them using the assumed similarity measure.

On the other hand, the "reward" attribute was not subjected to the classification procedure-it turned out to be impossible to clearly identify their names and (especially) weights, due to the great variety of rewards, varying time periods, or whether their number was limited. Moreover, there was practically no repeatability of rewards in projects. This problem is explained in more detail in Section 3 of this paper.

The second research hypothesis was also positively verified as subsequent classification procedures, the so-called cross-classification procedures, were carried out, and the obtained results allowed to demonstrate the strength of mutual correlation of attributes.

The aim of the article has been achieved for four out of the five crowdfunding attributes. It has been shown that the attributes can be classified using the presented procedure based on two parameters: closeness (similarity) and category weights.

Based on the research results included in the tables and class chains of project categories and their cross-comparisons, we can conclude the following:

- Project categories classified according to the selected attributes are combined into classes of different sizes, which means that they belong to different ranges of the 
attributes' values, and the number of classes within the attributes is similar (here: five and six).

- After carrying out the majority of the classification procedures, the weights assigned to the categories are no longer relevant, as they are assigned to different classes whose thematic scope is very wide.

- The correlation between the studied attributes was the highest in the tandem of profitability - campaign duration in two category classes, and the weakest in the tandem of the number of backers and campaign duration; the maximum correlation amounted to 0.4-0.7.

- The correlation was the weakest in the tandem of the number of supporters and duration of the campaign, as 14 pairs of classes show no correlation whatsoever.

One research limitation may be basing the analyses and classification of crowdfunding attributes on only one platform. Kickstarter is the leading platform due to the number of projects posted there and the number of successful projects, but each platform has its own specificity, subject, nomenclature and number of categories. It may result in the necessity to modify the input data before running the classification procedures according to the presented algorithm. The weaknesses of the conducted research include the omission of the "reward" attribute, which was explained in Section 3 of this article. However, it does not have any essential significance in relation to the general interpretation of the obtained results.

These research findings may prove useful in facilitating the decision-making process of all crowdfunding partners: crowdfunding platform owners, project owners and creators, and people considering backing certain projects, including strategic investors.

The prognoses for crowdfunding seem very promising (according to fundly.com), thus it is worth proposing future research directions for this phenomenon of social energy. They may concern the following, among others:

- Comparing and analyzing the specificity of small and large crowdfunding platforms,

- Analyzing crowdfunding models according to their various attributes,

- Changes and trends in creating project categories,

- The issue of rewards; the possibility of limiting them to a certain framework; and analyzing whether their various combinations and changes have the desired (financial) effect, whether rewards are so important to project backers that they should be offered in a certain form, or whether the backers are also lost in their analysis.

The authors of this paper intend to further study the fascinating world of crowdfunding with its many aspects, traps and victories, and, at the same time, we wish our dear colleagues good luck in discovering the secrets of sharing economy.

Author Contributions: Conceptualization, Z.S. and A.S.; methodology, A.S.; software, Z.S.; validation, Z.S.; formal analysis, Z.S. and A.S.; investigation, A.S.; resources, Z.S.; data curation, Z.S.; writing original draft preparation, Z.S. and A.S.; writing-review and editing, Z.S. and A.S.; visualization, Z.S. Both authors have read and agreed to the published version of the manuscript.

Funding: This research received no external funding.

Institutional Review Board Statement: Not applicable.

Informed Consent Statement: Not applicable.

Data Availability Statement: Webrobots.io/kickstarter-datasets-sources, data from 17 June 2021.

Conflicts of Interest: The authors declare no conflict of interest.

\section{References}

1. Smil, V. Energy in Nature and Society: General Energetics of Complex Systems; The MIT Press: Cambridge, MA, USA, 2008. [CrossRef]

2. Treimikiene, D. Externalities of power generation in Visegrad countries and their integration through support of renewables. Econ. Sociol. 2021, 14, 89-102. [CrossRef] 
3. Tvaronavičienè, M.; Prakapienè, D.; Garškaitè-Milvydienè, K.; Prakapas, R.; Nawrot, Ł. Energy Efficiency in the Long-Run in the Selected European Countries. Econ. Sociol. 2018, 11, 245-254. [CrossRef] [PubMed]

4. Streimikiene, D. Ranking of Baltic States on progress towards the main energy security goals of European energy union strategy. J. Int. Stud. 2020, 13, 24-37. [CrossRef] [PubMed]

5. Stavytskyy, A.; Kharlamova, G.; Giedraitis, V.; Šumskis, V. Estimating the interrelation between energy securi-ty and macroeconomic factors in European countries. J. Int. Stud. 2018, 11, 217-238. [CrossRef]

6. Shindina, T.; Streimikis, J.; Sukhareva, Y.; Nawrot, Ł. Social and Economic Properties of the Energy Markets. Econ. Sociol. 2018, 11, 334-344. [CrossRef]

7. Hnatyshyn, M. Decomposition analysis of the impact of economic growth on ammonia and nitrogen oxides emissions in the European Union. J. Int. Stud. 2018, 11, 201-209. [CrossRef] [PubMed]

8. Vo, H.D. Sustainable agriculture \& energy in the U.S.: A link between ethanol production and the acreage for corn. Econ. Sociol. 2020, 13, 259-268. [CrossRef]

9. Svazas, M.; Navickas, V.; Krajnakova, E.; Nakonieczny, J. Sustainable supply chain of the biomass cluster as a factor for preservation and enhancement of forests. J. Int. Stud. 2019, 12, 309-321. [CrossRef]

10. Block, J.; Hornuf, L.; Moritz, A. Which updates during an equity crowdfunding campaign increase crowd participation? Small Bus. Econ. 2018, 50, 3-27. [CrossRef]

11. Guenther, C.; Johan, S.; Schweizer, D. Is the crowd sensitive to distance?-How investment decisions differ by investor type. Small Bus. Econ. 2018, 50, 289-305. [CrossRef]

12. Polzin, F.; Toxopeus, H.; Stam, E. The wisdom of the crowd in funding: Information heterogeneity and social networks of crowdfunders. Small Bus. Econ. 2017, 50, 251-273. [CrossRef]

13. Ryoba, M.; Qu, S.; Ji, Y.; Qu, D. The Right Time for Crowd Communication during Campaigns for Sustainable Success of Crowdfunding: Evidence from Kickstarter Platform. Sustainability 2020, 12, 7642. [CrossRef]

14. Menor-Campos, A.; García-Moreno, M.D.L.B.; López-Guzmán, T.; Hidalgo-Fernández, A. Effects of Collaborative Economy: A Reflection. Soc. Sci. 2019, 8, 142. [CrossRef]

15. Rey-Martí, A.; Mohedano-Suanes, A.; Simón-Moya, V. Crowdfunding and Social Entrepreneurship: Spotlight on Intermediaries. Sustainability 2019, 11, 1175. [CrossRef]

16. Van Montfort, K.; Siebers, V.; De Graaf, F.J. Civic Crowdfunding in Local Governments: Variables for Success in the Netherlands? J. Risk Financ. Manag. 2020, 14, 8. [CrossRef]

17. Vismara, S. Information Cascades among Investors in Equity Crowdfunding. Entrep. Theory Pract. 2018, 42, 467-497. [CrossRef]

18. Alegre, I.; Moleskis, M. Beyond financial motivations in crowdfunding: A systematic literature review of donations and rewards. Voluntas 2021, 32, 276-287. [CrossRef]

19. Chen, Y.; Dai, R.; Yao, J.; Li, Y. Donate Time or Money? The Determinants of Donation Intention in Online Crowdfunding. Sustainability 2019, 11, 4269. [CrossRef]

20. Le Pendeven, B.; Bardon, T.; Manigart, S. Explaining Academic Interest in Crowdfunding as a Research Topic. Br. J. Manag. 2021. [CrossRef]

21. Butticè, V.; Ughetto, E. What, Where, Who, and How? A Bibliometric Study of Crowdfunding Research. IEEE Trans. Eng. Manag. 2021. [CrossRef]

22. Heidingsfelder, J.; Beckmann, M. A governance puzzle to be solved? A systematic literature review of fragmented sustainability governance. Manag. Rev. Q. 2020, 70, 355-390. [CrossRef]

23. Testa, S.; Nielsen, K.R.; Bogers, M.; Cincotti, S. The role of crowdfunding in moving towards a sustainable society. Technol. Forecast. Soc. Chang. 2019, 141, 66-73. [CrossRef]

24. Motylska-Kuzma, A. Crowdfunding and Sustainable Development. Sustainability 2018, 10, 4650. [CrossRef]

25. Martínez-Climent, C.; Costa-Climent, R.; Oghazi, P. Sustainable Financing through Crowdfunding. Sustainability 2019, 11, 934. [CrossRef]

26. Wehnert, P.; Baccarella, C.V.; Beckmann, M. In crowdfunding we trust? Investigating crowdfunding success as a signal for enhancing trust in sustainable product features. Technol. Forecast. Soc. Chang. 2019, 141, 128-137. [CrossRef]

27. Liang, X.; Hu, X.; Jiang, J. Research on the Effects of Information Description on Crowdfunding Success within a Sustainable Economy-The Perspective of Information Communication. Sustainability 2020, 12, 650. [CrossRef]

28. Konhäusner, P. Crowdsourcing in Sustainable Retail—A Theoretical Framework of Success Criteria. J. Risk Financ. Manag. 2021, 14, 87. [CrossRef]

29. Jelinčić, D.; Šveb, M. Financial Sustainability of Cultural Heritage: A Review of Crowdfunding in Europe. J. Risk Financ. Manag. 2021, 14, 101. [CrossRef]

30. Rossi, A.; Vismara, S.; Meoli, M. Voting rights delivery in investment-based crowdfunding: A cross-platform analysis. J. Ind. Bus. Econ. 2019, 46, 251-281. [CrossRef]

31. Dilger, M.G.; Jovanović, T.; Voigt, K.I. Upcrowding energy co-operatives-evaluating the potential of crowd-funding for business model innovation of energy co-operatives. J. Environ. Manag. 2017, 198, 50-62. [CrossRef]

32. Wallmeroth, J.; Wirtz, P.; Groh, A.P. Venture Capital, Angel Financing, and Crowdfunding of Entrepreneurial Ventures: A Literature Review. Found. Trends Entrep. 2018, 14, 1-129. [CrossRef]

33. Vismara, S. Equity retention and social network theory in equity crowdfunding. Small Bus. Econ. 2016, 46, 579-590. [CrossRef] 
34. Chu, C.-C.; Cheng, Y.-F.; Tsai, F.-S.; Tsai, S.-B.; Lu, K.-H. Open Innovation in Crowdfunding Context: Diversity, Knowledge, and Networks. Sustainability 2019, 11, 180. [CrossRef]

35. Miglo, A. Financing of Entrepreneurial Firms in Canada: Some Patterns. Adm. Sci. 2020, 10, 50. [CrossRef]

36. Rossi, A.; Vismara, S. What do crowdfunding platforms do? A comparison between investment-based platforms in Europe. Eurasian Bus. Rev. 2017, 8, 93-118. [CrossRef]

37. Yu, Z.; Hafeez, M.; Liu, L.; Mahmood, M.T.; Wu, H. Evaluating the Minor Coarse Cereals Product Crowdfunding Platform through Evolutionary Game Analysis. Sustainability 2019, 11, 1299. [CrossRef]

38. Kang, H.; Kim, H.U. Who Can Survive in an ICT-Enabled Crowdfunding Platform? Sustainability 2020, 12, 504. [CrossRef]

39. Flórez-Parra, J.M.; Martín, G.R.; Serrano, C.R. Corporate Social Responsibility and Crowdfunding: The Experience of the Colectual Platform in Empowering Economic and Sustainable Projects. Sustainability 2020, 12, 5251. [CrossRef]

40. Bretschneider, U.; Leimeister, J.M. Not just an Ego trip: Exploring Backers' motivation for funding in incentive-based crowdfunding. J. Strateg. Inf. Syst. 2017, 26, 246-260. [CrossRef]

41. Pérez, L.P.Y.; Egea, P. About Intentions to Donate for Sustainable Rural Development: An Exploratory Study. Sustainability 2019, 11, 765. [CrossRef]

42. Li, Y.; Zhang, Z.; Wang, R.; Chen, Y. Consumer Purchase Intention toward Crowdfunding Products/Services: A Cost-Benefit Perspective. Sustainability 2019, 11, 3579. [CrossRef]

43. Kim, M.; Hall, C. Can Co-Creation and Crowdfunding Types Predict Funder Behaviour? An Extended Model of Goal-Directed Behaviour. Sustainability 2019, 11, 706. [CrossRef]

44. Miglo, A. Crowdfunding in a Competitive Environment. J. Risk Financ. Manag. 2020, 13, 39. [CrossRef]

45. Seo, B.; Park, D. Did You Invest Less Than Me? The Effect of Other's Share of Investment on Psychological Ownership of Crowdfunding Projects. Sustainability 2020, 12, 202. [CrossRef]

46. Song, Y.; Tian, X. Managerial Responses and Customer Engagement in Crowdfunding. Sustainability 2020, 12, 3389. [CrossRef]

47. Rodriguez-Ricardo, Y.; Sicilia, M.; López, M. Altruism and Internal Locus of Control as Determinants of the Intention to Participate in Crowdfunding: The Mediating Role of Trust. J. Theor. Appl. Electron. Commer. Res. 2019, 14, 1-16. [CrossRef]

48. Alharbey, M.; Van Hemmen, S. Investor Intention in Equity Crowdfunding. Does Trust Matter? J. Risk Financ. Manag. 2021, 14, 53. [CrossRef]

49. Khurana, I. Legitimacy and Reciprocal Altruism in Donation-Based Crowdfunding: Evidence from India. J. Risk Financ. Manag. 2021, 14, 194. [CrossRef]

50. Mohammadi, A.; Shafi, K. Gender differences in the contribution patterns of equity-crowdfunding investors. Small Bus. Econ. 2017, 50, 275-287. [CrossRef]

51. Wasiuzzaman, S.; Lee, C.; Boon, O.; Chelvam, H. Examination of the Motivations for Equity-Based Crowdfunding in an Emerging Market. J. Theor. Appl. Electron. Commer. Res. 2021, 16, 63-67. [CrossRef]

52. Kukk, M.-L.; Laidroo, L. Institutional Drivers of Crowdfunding Volumes. J. Risk Financ. Manag. 2020, 13, 326. [CrossRef]

53. Kim, M.; Hall, C.; Han, H. Behavioural Influences on Crowdfunding SDG Initiatives: The Importance of Personality and Subjective Well-Being. Sustainability 2021, 13, 379. [CrossRef]

54. Yang, X.; Zhao, K.; Tao, X.; Shiu, E. Developing and Validating a Theory-Based Model of Crowdfunding Investment Intention -Perspectives from Social Exchange Theory and Customer Value Perspective. Sustainability 2019, 11, 252. [CrossRef]

55. Dikaputra, R.; Sulung, L.A.K.; Kot, S. Analysis of Success Factors of Reward-Based Crowdfunding Campaigns Using Multi-Theory Approach in ASEAN-5 Countries. Soc. Sci. 2019, 8, 293. [CrossRef]

56. Comeig, I.; Mesa-Vázquez, E.; Sendra-Pons, P.; Urbano, A. Rational Herding in Reward-Based Crowdfunding: An MTurk Experiment. Sustainability 2020, 12, 9827. [CrossRef]

57. Usman, S.; Bukhari, F.; You, H.; Badulescu, D.; Gavrilut, D. The Effect and Impact of Signals on Investing Decisions in RewardBased Crowdfunding: A Comparative Study of China and the United Kingdom. J. Risk Financ. Manag. 2020, 13, 32. [CrossRef]

58. Sauermann, H.; Franzoni, C.; Shafi, K. Crowdfunding scientific research: Descriptive insights and correlates of funding success. PloS ONE 2019, 14, e0208384. [CrossRef]

59. Hörisch, J. Crowdfunding for environmental ventures: An empirical analysis of the influence of environmental orientation on the success of crowdfunding initiatives. J. Clean. Prod. 2015, 107, 636-645. [CrossRef]

60. Ter Wal, A.L.J.; Alexy, O.; Block, J.; Sandner, P.G. The best of both worlds the benefits of open-specialized and closed-diverse syndication networks for new ventures' success. Adm. Sci. Q. 2016, 61, 393-432. [CrossRef]

61. Usman, S.M.; Bukhari, F.A.S.; Usman, M.; Badulescu, D.; Sial, M.S. Does the Role of Media and Founder's Past Success Mitigate the Problem of Information Asymmetry? Evidence from a UK Crowdfunding Platform. Sustainability 2019, 11, 692. [CrossRef]

62. Ullah, S.; Zhou, Y. Gender, Anonymity and Team: What Determines Crowdfunding Success on Kickstarter. J. Risk Financial Manag. 2020, 13, 80. [CrossRef]

63. Fernandez-Blanco, A.; Villanueva-Balsera, J.; Rodriguez-Montequin, V.; Moran-Palacios, H. Key Factors for Project Crowdfunding Success: An Empirical Study. Sustainability 2020, 12, 59. [CrossRef]

64. Schraven, E.; van Burg, E.; van Gelderen, M.; Masurel, E. Predictions of Crowdfunding Campaign Success: The Influence of First Impressions on Accuracy and Positivity. J. Risk Financ. Manag. 2020, 13, 33. [CrossRef]

65. Venturelli, V.; Pedrazzoli, A.; Gallo, G. Birds of a Feather Flock Together: The Inclusive Effect of Similarity Patterns in Equity Crowdfunding. Sustainability 2020, 12, 353. [CrossRef] 
66. Chen, L. Investigating the Impact of Competition and Incentive Design on Performance of Crowdfunding Projects: A Case of Independent Movies. J. Theor. Appl. Electron. Commer. Res. 2021, 16, 791-810. [CrossRef]

67. von Selasinsky, C.; Lutz, E. The Effects of Pro-Social and Pro-Environmental Orientation on Crowdfunding Performance. Sustainability 2021, 13, 606. [CrossRef]

68. Batley, S. Classification in Theory and Practice; Chandos Publishing: Amsterdam, The Netherlands, 2005. [CrossRef]

69. Ishibuchi, H.; Nakashima, T.; Nii, M. Classification and Modeling with Linguistic Information Granules: Advanced Approaches to Linguistic Data Mining; Springer: Berlin/Heidelberg, Germany, 2004. [CrossRef]

70. Sauerwein, C.; Pekaric, I.; Felderer, M.; Breu, R. An analysis and classification of public information security data sources used in research and practice. Comput. Secur. 2019, 82, 140-155. [CrossRef]

71. Broughton, V. The need for a faceted classification as the basis of all methods of information retrieval. Aslib Proc. 2006, 58, 49-72. [CrossRef]

72. Caroll, J.; Roeloffs, R. Computer Selection of Keywords Using Word-Frequency Analysis. Am. Doc. 1969, 20, 227-233. [CrossRef]

73. Rohhio, J.J. Document Retrieval Systems-Optimization and Evaluation; Report No. ISR 10; National Science Foundation, Harvard Computation Laboratorium: Cambridge, MA, USA, 1966.

74. Hill, D.R. A Vector Clustering Technique. In Mechanized Information Storage. Retrieval and Dissemination, Proceeding of the FID/IFIP Joint Conference, Rome, Italy, 14-17 June 1967; North Holland Publishing Company: Amsterdam, NL, USA, 1968.

75. Vaswani, P.K.T. A Technique for Cluster Emphasis and its Applications to Automatic Indexing. In Information Processing, Proceedings of IFIP Congress 1968, Edinburgh, UK, 5-10 August 1968; Macmillan and Co.: London, UK, 1968 ; Volume 2.

76. Dattola, R.T. Experiment with a Fast Algorithm for Automatic Classification. In The SMART System-Experiments in Automatic Document Processing; Salton, G., Ed.; Prentice-Hall: Englewood Cliffs, NJ, USA, 1971.

77. Dattola, R.T. A Fast Algorithm for Automatic Classification; Report No. ISR-14 to the National Science Foundation, Section V; Department Computer Science, Cornell University: Ithaca, NY, USA, 1968.

78. Gitman, I.; Levine, M.D. An Algorithm for Detecting Unimodal Fuzzy Sets and Its Application as a Cliustering Technique. IEEE Trans. Comput. 1970, 100, 583-593. [CrossRef] 